\title{
Endophytic Fungi from Four Indonesian Medicinal Plants andTheir Inhibitory Effect on Plant Pathogenic Fusarium oxysporum
}

\author{
Nampiah Sukarno ${ }^{1 *}$, Rohani Cinta Badia Ginting ${ }^{1,2}$, Utut Widyastuti ${ }^{\dagger 1,3}$, Latifah Kosim Darusman ${ }^{\dagger 4,5}$, Shigehiko \\ Kanaya $^{6}$, Irmanida Batubara ${ }^{4,5}$, I Nyoman Pugeg Aryantha, ${ }^{7,8}$, Mashuri Waite ${ }^{9}$ \\ ${ }^{1}$ Department of Biology, Faculty of Mathematics and Natural Sciences, IPB University, Darmaga Campus, Bogor, Indonesia \\ ${ }^{2}$ Indonesian Soil Research Institute, Bogor, Indonesia \\ ${ }^{3}$ Research Center for Bioresources and Biotechnology, IPB University, Darmaga Campus, Bogor, Indonesia \\ ${ }^{4}$ Department of Chemistry, Faculty of Mathematics and Natural Sciences, IPB University, Darmaga Campus, Bogor, Indonesia \\ ${ }^{5}$ Tropical Biopharmaca Research Center, IPB University, Bogor, Indonesia \\ ${ }^{6}$ Graduate School of Information Science, Nara Institute of Science and Technology, Takayama, Ikoma, Nara, Japan \\ ${ }^{7}$ School of Life Sciences and Technology, Institut Teknologi Bandung, Bandung, Indonesia \\ ${ }^{8}$ Research Center for Biosciences and Biotechnology, Institut Teknologi Bandung, Bandung, Indonesia \\ ${ }^{9}$ Gill Ewa Lands, Honolulu, Hawaii, USA
}

\section{ARTICLE INFO}

Article history:

Received October 15, 2020

Received in revised form February 15, 2021

Accepted March 1, 2021

\section{KEYWORDS:}

Antagonistic activity,

Biodiversity,

Endophytic fungi,

ITS rDNA,

Phylogenetic analysis

\begin{abstract}
The medicinal plants Centella asiatica, Curcuma xanthorrhiza, Guazuma ulmifolia, and Hydrocotyle verticillata are widely used in Indonesian traditional medicine, but little is known about their associated endophytic fungi. This research aimed to study the diversity of endophytic fungi derived from functional parts of these plants and to evaluate their potential as antifungal agents against the plant pathogenic fungus Fusarium oxysporum. A total of 17 isolates of endophytic fungi were obtained: nine from leaves of $G$. ulmifolia, three each from leaves of $C$. asiatica and $H$. verticillata, and two from rhizomes of $C$. xanthorrhiza. The genus Colletotrichum was found in all plants studied, but each plant was associated with different species. Colletotrichum aeschynomenes was associated with $C$. xanthorrhiza, $C$. siamense was associated with $C$. asiatica, and $C$. tropicale was associated with $G$. ulmifolia and $H$. verticillata. The species Curvularia affinis, Diaporthe tectonae, Lasiodiplodia mahajangana, Parengyodontium album, Talaromyces trachyspermus, and Speiropsis pedatospora were found only in G. ulmifolia; while Didymella coffeae-arabicae and Muyocopron laterale were found only in $H$. verticillata. The endophytic fungi showed inhibition activity against $F$. oxysporum with inhibition values of 6.0-78.9\%, T. trachyspermus JBd10 and $C$. affinis JBd14 gave the highest inhibition activity.
\end{abstract}

\section{Introduction}

The medicinal plants Centella asiatica L. (asiatic pennywort), Curcuma xanthorrhiza Roxb. (java turmeric), Guazuma ulmifolia Lamk. (bay cedar), and Hydrocotyle verticillata Thunb. (whorled pennywort) are well known for their usage and medicinal properties. The raw material for medicines can be obtained from different parts of a medicinal plant based on their various active ingredients. The dried sample can be obtained from a particular part of the medicinal plant such as the leaf, stem, rhizome, or root that contains a high concentration of the active

\footnotetext{
* Corresponding Author

E-mail Address: nampiah@apps.ipb.ac.id
}

compound, or from the whole plant. In traditional medicines, Curcuma dried sample is derived from rhizomes, while Centella, Guazuma, and Hydrocotyle dried samples are derived from leaves.

Endophytes are microorganisms that live within plant tissues for at least part of their life cycle without causing apparent disease. Fungi and bacteria are the most common microbes living as endophytes, but the most commonly isolated are fungi (Hardoim et al. 2015). There are relatively few reports on the endophytic fungi associated with medicinal plants. Among them, Hammerschmidt et al. (2015) isolated Xylaria sp. from healthy leaves of plants collected on the island of Timor, Indonesia, and the fungus produced a new compound (resacetophenone). Septiana et al. (2017) successfully isolated eleven 
endophytic fungi from the turmeric plant, some of which showed antibacterial and anti-histamine activities. Endophytic fungi reported for $C$. asiatica included Colletotrichum higginsianum, Guignardia mangiferae, and Glomerella cingulata (Rakotoniriana et al. 2008). Penicillium sp. derived from turmeric leaves produced alkaloids, phenols, flavonoids, tannin, glycosides, and cellulase enzyme (Devi et al. 2012). Endophytic fungi associated with G. ulmifolia were Muscodor albus (Strobel et al. 2007), Pestalotiopsis sp. (Russell et al. 2011), and Nigrograna mackinnonii, which produced limonene compound (Shaw et al. 2015). Strobel et al. (2007) reported that Muscodor albus E-6 obtained from G. ulmifolia branches could produce many secondary metabolites. So far, there are no reports of endophytic fungi associated with the $H$. verticillata plant.

Endophytic fungi are widely accepted to be able to synthesize bioactive natural compounds (Strobel and Daisy 2003), including compounds with antimicrobial activity for defense against pathogens (Kusari et al. 2013), which may be used as biocontrol agents in agriculture. Endophytic fungi inhibit the growth or stop the reproduction of pathogens by many mechanisms such as antagonism, mycoparasitism, antibiosis, and competition (Cook 1993). They produce bioactive compounds in two ways, either from precursors initiated by their metabolism or from precursors produced by the plant's metabolism. This research aimed to obtain endophytic fungi from the medicinally used organs of C. asiatica, C. xanthorrhiza, G. ulmifolia, and $H$. verticillata; to study their diversity in these organs; and to evaluate their antifungal activity against the plant pathogenic fungus Fusarium oxysporum.

\section{Materials and Methods}

\subsection{Plant Materials}

Endophytic fungi were isolated from the medicinally used organs of $C$. asiatica, C. xanthorrhiza, G. ulmifolia, and $H$. verticillata. All the medicinal plant samples were obtained from the living collections of the Indonesian Medicinal and Aromatic Crops Research Institute, Bogor, Indonesia. In traditional Indonesian remedies, the medicinally used organs of $C$. asiatica, G. ulmifolia, and $H$. verticillata are the leaves, while for $C$. xanthorrhiza are the rhizomes.

Five clumps of fresh and healthy plants of $C$. asiatica, G. ulmifolia, and $H$. verticillata were randomly selected and harvested by cutting the three leaves from the third to fifth leaf from the top of the plants. For C. xanthorrhiza, three clumps of fresh and healthy plants were carefully harvested by digging up the plant and cutting the rhizomes. Samples were then put in clean plastic bags, transported to the laboratory, and processed within 24 hours of collection. Each sample was washed thoroughly with running tap water and followed up by rinsing with sterilized reverse osmosis water three times and pooling them to make a composite sample.

\subsection{Isolation of Endophytic Fungi}

All preparations and isolation processes were carried out in a biosafety cabinet. Leaf samples were cut into small pieces of $2 \times 2 \mathrm{~cm}^{2}$ size. The rhizomes of $C$. xanthorrhiza were peeled and then cut into $2 \mathrm{x}$ $2 \times 2 \mathrm{~cm}^{3}$ pieces. Surface sterilization was conducted by immersing the sample in $70 \%$ ethanol for $1 \mathrm{~min}$, soaking in $0.5 \%$ hypochlorite solution for $5 \mathrm{~min}$, and $70 \%$ ethanol for $1 \mathrm{~min}$, and finally washing with sterilized distilled water six times. Then the samples were blotted on sterile Whatman filter paper for 12 hours. Four pieces from each cutting sample were randomly chosen and cultured on potato dextrose agar (PDA, difco) plates containing rose bengal (30 $\left.\mathrm{mg} \mathrm{L}^{-1}\right)$ and chloramphenicol ( $0.5 \mathrm{~g}$ $\left.\mathrm{L}^{-1}\right)$. Media plates were sealed and incubated at $28^{\circ} \mathrm{C}$ over 21 days, during which time they were checked daily for hyphal growth (Hallmann et al. 2007). The hyphal tips arising from the colonies having different characteristics were picked and transferred onto new PDA plates without being supplemented with either rose bengal or chloramphenicol. Each fungal isolate was purified to obtain a single colony.

\subsection{Identification of Endophytic Fungal Isolates}

The pure isolates having different characteristics were identified by a combination of morphological characteristics (Barnett and Hunter 1998) and molecular analyses. DNA extraction was prepared according to the CTAB-based extraction method (Sambrook and Russel 2000). The fungi were cultured in potato dextrose broth (PDB, difco) and incubated in a shaker at $120 \mathrm{rpm}$ $28^{\circ} \mathrm{C}$ for seven days. Mycelia were harvested through sterilized filter paper by vacuum filtration. The mycelia were frozen in liquid nitrogen and ground in a sterile mortar. About $0.5 \mathrm{~g}$ of mycelia powder was mixed with warm extraction buffer $(600 \mu \mathrm{l}$ PVP and $1.2 \mu \mathrm{l}$ 
$\mathrm{CTAB}$ ) in a 1.5-ml Eppendorf tube. It was inverted and incubated at $65^{\circ} \mathrm{C}$ for $30 \mathrm{~min}$. The tube was then incubated on ice for $5 \mathrm{~min}$ and then $600 \mu \mathrm{l}$ of a mixed solution of chloroform: alcohol (24:1) was added. It was then inverted and centrifuged for $10 \mathrm{~min}$ at $10^{\circ} \mathrm{C}$, $25,000 \times g$. The aqueous phase was removed carefully to a new tube, then added with an equal volume of phenol: chloroform: isoamyl alcohol (25:24:1). The tube was inverted and centrifuged again for $5 \mathrm{~min}$ at $4^{\circ} \mathrm{C}, 25,000 \times \mathrm{g}$. The supernatant was mixed with an equal volume of $2 \mathrm{M} \mathrm{NaOAc} \mathrm{pH} 5.2$ and $2 \mathrm{x}$ volume of cold $\mathrm{EtOH}$ in a new tube, and incubated for $30 \mathrm{~min}$ at $20^{\circ} \mathrm{C}$, and then centrifuged at $25,000 \times \mathrm{g}$, at $4^{\circ} \mathrm{C}$ for $30 \mathrm{~min}$. DNA pellets were collected and washed with $500 \mu \mathrm{l} 70 \%$ cold ethanol and then centrifuged for 5 $\min$ at $4^{\circ} \mathrm{C}, 25,000 \times \mathrm{g}$. DNA pellets were dried briefly using a vacuum, resuspended in $20 \mu$ of sterilized double-distilled water, added with $0.2 x$ volume of RNAse, and incubated for $10 \mathrm{~min}$ at $37^{\circ} \mathrm{C}$. The DNA was then incubated for $10 \mathrm{~min}$ at $70^{\circ} \mathrm{C}$ to inactivate the RNAse. Fungal DNA was then stored in a freezer until used.

The DNA was then subjected to PCR amplification using the universal primers pair of ITS1 (forward) (5'-TCC GTA GGT GAA CCT GCG G-3') and ITS4 (reverse) (5'-TCC TCC GCT TAT TGA TAT GC-3') (White et al. 1990). The amplified fragments consisted of the internal transcribed spacer (ITS) regions of the extracted DNA, including the 5.8S rDNA. The PCR reaction was performed in a $60 \mu$ reaction mixture which consisted of $42.6 \mu \mathrm{l}$ sterilized ddH2O, $6 \mu$ l buffer (10x), $1.2 \mu \mathrm{l} 2 \mathrm{mM}$ dNTP, $1.5 \mu \mathrm{l} 10 \mathrm{pmol}$ of each forward and reverse primer, 1.2 $\mu l 5$ U Taq DNA polymerase, and $6 \mu$ DNA template. The PCR amplification reaction was carried out under the following conditions: initial denaturation for 5 min at $95^{\circ} \mathrm{C}$, followed by 35 cycles of denaturation, annealing, and extension at $72^{\circ} \mathrm{C}$ for $1 \mathrm{~min}, 94^{\circ} \mathrm{C}$ for 30 seconds, and $52^{\circ} \mathrm{C}$ for 30 seconds, respectively. This process was followed by a final re-extension step of $72^{\circ} \mathrm{C}$ for $5 \mathrm{~min}$ and finally stored at $25^{\circ} \mathrm{C}$ for 10 min using a Gene Amp 9700 thermal cycler (Applied Biosystems, USA).

The PCR products were purified and sequenced by First Base (Malaysia) using the same primers. The sequence was analyzed using the BioEdit Ver.7 (Hall 1999) and aligned using Clustal W (Thompson et al. 1994). The sequence similarity was determined by using available DNA fungal sequences at MycoBank (https://www.mycobank.org) and GenBank (BLAST) (https://blast.ncbi.nlm.nih.gov/Blast.cgi). Phylogenetic analyses were conducted using Maximum Likelihood methods in MEGA6 (Tamura et al. 2013). The reference GeneBank accession was used to construct the phylogenetic tree of Aspergillus based on Samson et al. (2014), Colletotrichum based on Cannon et al. (2012), Curvularia based on Marin-Felix et al. (2020), Diaporthe based on Dissanayake et al. (2017), Didymella based on Scarpari et al. (2020) and Chen et al. (2017), Neocosmospora based on Sandoval-Denis and Crous (2018), Lasiodiplodia based on Abdollahzadeh et al. (2010), Parengyodontium based on Tsang et al. (2016), and Talaromyces based on Adhikari et al. (2015). Sequences used for phylogenetic analysis are shown in Table 1. The maximum likelihood tree was constructed using the best DNA model (Nei and Kumar 2000). The genera Aspergillus, Colletotrichum, Curvularia, Neocosmospora, and Lasiodiplodia used the $\mathrm{K} 2+\mathrm{G}$ model; Diaporthe used the $\mathrm{K} 2+\mathrm{G}+\mathrm{I}$ model; Parengyodontium used the T92+G model; and the genera Didymella and Talaromyces used the $\mathrm{T} 92+\mathrm{G}+\mathrm{I}$ (G Gamma distributed, I evolutionarily invariable, K2 Kimura 2-parameter, T92 Tamura 3-parameter). Phylogenetic analyses of the genera Muyocopron and Speiropsis were not done due to the limited availability of DNA sequences in the database. Gaps and missing data were treated as complete deletions. Initial trees for ML were made by the $\mathrm{NJ} /$ BioNJ algorithm and the branch swap filter was set very strong. Support for specific nodes on the ML tree was estimated by bootstrapping 1,000 replications. The nucleotide sequences generated in this study were deposited in GenBank (https://www. ncbi.nlm.nih.gov/).

\subsection{In Vitro Antagonistic Bioassay}

The antagonistic activities of the endophytic fungi isolates were evaluated against the plant pathogenic fungus $F$. oxysporum (IPBCC.88.0.12 or CBS 254.52) using the antagonist assays method of Morton and Stroube (1955) by using a dual culture technique in vitro assay on PDA. First, the endophytic fungi isolates and pathogenic fungus $F$. oxysporum were grown on separate PDA plates for seven days at $28^{\circ} \mathrm{C}$. A $5 \mathrm{~mm}$ diameter mycelial plug of endophytic fungus was placed $1 \mathrm{~cm}$ away from the periphery at one end of a Petri dish containing PDA and incubated at room temperature for 4 days. A $5 \mathrm{~mm}$ in diameter culture plug of $F$. oxysporum was placed in the same Petri dish at a distance of $1 \mathrm{~cm}$ from the edge, at the opposite side to the endophytic fungus. In the control treatment, an agar disc $(5 \mathrm{~mm}$ in diameter) was placed $1 \mathrm{~cm}$ away from the periphery 
Table 1. DNA sequence accession numbers of the isolates included in this study

Species Isolates ITS GenBank accession numbers

Sources

DNA sequence accession numbers for phylogenetic analysis of Aspergillus

\begin{tabular}{|c|c|c|c|}
\hline Aspergillus aculeatus & NRRL 5094 & EF661221 & Soil \\
\hline A. avenaceus & CBS $109.46^{\mathrm{NT}}$ & AF104446 & Pisum sativum seed \\
\hline A. calidoustus & CBS $121601^{\mathrm{T}}$ & HE616558 & Bronchoalveolar lavage specimen \\
\hline A. clavatus & NRRL $1^{\mathrm{T}}$ & EF669942 & Soil \\
\hline A. fischeri & NRRL $181^{\text {NT }}$ & EF669936 & Canned apples \\
\hline A. flavipes & NRRL 302 LT & EF669591 & Soil \\
\hline A. flavus & NRRL $1957^{\mathrm{NT}}$ & AF027863 & Moldy cellophane \\
\hline A. fumigatus & NRRL $163^{\mathrm{T}}$ & EF669931 & Chicken lung \\
\hline A. funiculosus & NRRL $4744^{\mathrm{T}}$ & EF661223 & Soil \\
\hline A. glaucus & NRRL $116^{\mathrm{NT}}$ & EF652052 & House lumber \\
\hline A. montevidensis & NRRL $108^{\mathrm{NT}}$ & EF652077 & Tympanic membrane \\
\hline A. nidulans & NRRL $187^{\mathrm{NT}}$ & EF652427 & Soil \\
\hline A. ochraceus & NRRL $398^{\mathrm{NT}}$ & EF661419 & Unknown \\
\hline A. penicillioides & NRRL $4548^{\mathrm{NT}}$ & EF652036 & Human skin \\
\hline \multirow{2}{*}{ A. pseudoterreus } & NRRL $4017^{\text {HT }}$ & NR_137472 & Soil \\
\hline & IPBCC 11.758 & SUB̈9428972 & Rhizomes of Curcuma xanthorrhiza \\
\hline A. sparsus & NRRL $1933^{\text {LT }}$ & EF661181 & Soil \\
\hline A. terreus & NRRL $255^{\mathrm{T}}$ & EF669586 & Soil \\
\hline A. togoensis & CBS $272.89^{\mathrm{T}}$ & AJ874113 & Seed \\
\hline \multirow[t]{3}{*}{ A. versicolor } & NRRL 238 ${ }^{\mathrm{NT}}$ & EF652442 & Unknown \\
\hline & IPBCC 11.760 & SUB9403371 & Leaves of Centella asiatica \\
\hline & IPBCC 11.749 & SUB9427202 & Leaves of Guazuma ulmifolia \\
\hline Talaromyces flavus & CBS $310.38^{\mathrm{T}}$ & JN899360 & Unknown \\
\hline
\end{tabular}

DNA sequence accession numbers for phylogenetic analysis of Colletotrichum

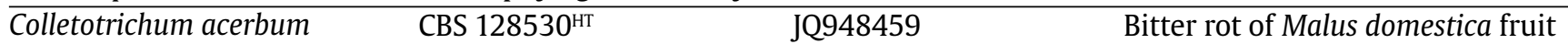

C. aeschynomenes

C. anthrisci

C. boninense

C. cliviae

C. curcumae

C. dracaenophilum

C. fructi

C. jasminigenum

C. kahawae

C. lindemuthianum

C. lineola

C. orbiculare

C. pseudoacutatum

C. pyricola

C. rhombiforme

C. siamense

C. torulosum

C. tropicale

C. truncatum

C. yunnanense

Monilochaetes infuscans
ICMP $17673^{\mathrm{HT}} \quad$ JX010176

IPBCC 11.757 SUB9432747

CBS $125334^{\mathrm{T}} \quad$ GU227845

CBS $123755^{\mathrm{HT}}$ AB051400

CBS $125375^{\mathrm{HT}}$ JX519223

IMI 288937ЕT GU227893

CBS $118199^{\mathrm{HT}}$ JX519222

CBS 346.37

MFLUCC 10-0273 ${ }^{\mathrm{HT}}$

ICMP $17816^{\mathrm{HT}}$

CBS $144.31^{\mathrm{ET}}$

CBS $125337^{\mathrm{ET}}$

CBS $514.97^{\mathrm{HT}}$

CBS $436.77^{\mathrm{HT}}$

CBS $128531^{\mathrm{HT}}$

CBS $129953^{\mathrm{HT}}$

ICMP $18578^{\mathrm{T}}$

IPBCC 13.1092

CBS $128544^{\mathrm{HT}}$

ICMP $18653^{\mathrm{HT}}$

IPBCC 11.752

IPBCC 11.747

CBS $151.35^{\mathrm{ET}}$

CBS $132135^{\mathrm{HT}}$

CBS 869.96
GU227844

HM131513

JX010231

JQ005779

GU227829

JQ005778

JQ948480

JQ948445

JQ948457

FJ972613

SUB9446440

JQ005164

GU994331

SUB9446618

SUB9433263

GU227862

EF369490

GU180626
Aeschynomene virginica

Rhizomes of Curcuma xanthorrhiza

Anthriscus sylvestris

Crinum asiaticum var. sinicum

Clivia miniata

Curcuma longa

Dracaena sanderana

Malus sylvestris

Jasminum sambac

Coffea arabica

Phaseolus vulgaris

Apiaceae, dead stem

Cucumis sativus

Pinus radiata

Pyrus communis, fruit rot

Olea europaea

Coffea arabica

Leaves of Centella asiatica

Solanum melongena

Theobroma cacao

Leaves of Hydrocotyle verticillata

Leaves of Guazuma ulmifolia

Phaseolus lunatus

Buxus sp.

Ipomoea batatas 
Table 1. Continued

\begin{tabular}{|c|c|c|c|}
\hline \multirow{2}{*}{\multicolumn{4}{|c|}{$\begin{array}{lcc}\text { Species } & \text { Isolates } & \text { ITS GenBank accession numbers } \\
\text { DNA sequence accession numbers for phylogenetic analysis of Curvularia }\end{array}$}} \\
\hline & & & \\
\hline \multirow{2}{*}{ Curvularia affinis } & CBS 154.34 & KJ909780 & Unknown \\
\hline & IPBCC 13.1088 & SUB9445050 & Leaves of Guazuma ulmifolia \\
\hline C. asiatica & MFLUCC $10-0711^{\mathrm{T}}$ & JX256424 & Panicum sp. \\
\hline C. beerburrumensis & BRIP $12942^{\mathrm{T}}$ & MH414894 & Eragrostis bahiensis \\
\hline C. cactivora & CBS 580.74 & MN688803 & Member of Cactaceae \\
\hline C. chiangmaiensis & СРC $28829^{\mathrm{T}}$ & MF490814 & Zea mays \\
\hline C. coicis & CBS $192.29^{\text {SynT }}$ & JN192373 & Coix lacryma \\
\hline C. crassiseptata & CBS 503.90 & LT631310 & Plant material \\
\hline C. cymbopogonis & CBS 419.78 & HG778985 & Yucca sp. \\
\hline C. dactyloctenii & BRIP $12846^{\mathrm{T}}$ & KJ415545 & Dactyloctenium radulans \\
\hline C. ellisii & CBS $193.62^{\mathrm{T}}$ & JN192375 & Air \\
\hline C. eragrosticola & BRIP $12538^{\mathrm{HT}}$ & MH414899 & Eragrostis pilosa \\
\hline C. gladioli & CBS 210.79 & HG778987 & Gladiolus sp. \\
\hline C. heteropogonis & CBS $284.91^{\mathrm{T}}$ & JN192379 & Heteropogon contortus \\
\hline C. intermedia & CBS 334.64 & HG778991 & Avena versicolor \\
\hline C. ischaemi & CBS $630.82^{\mathrm{T}}$ & JX256428 & Ischaemum indicum \\
\hline C. microspore & GUCC $6272^{\mathrm{T}}$ & MF139088 & Hippeastrum striatum leaf spot \\
\hline C. mosaddeghii & IRAN $3131^{\mathrm{cT}}$ & MG846737 & Syzygium cumini leaf spot \\
\hline C. neergaardii & BRIP 12919'soT & KJ415550 & Oryza sativa \\
\hline C. nodosa & CPC $28800^{\mathrm{T}}$ & MF490816 & Digitaria ciliaris \\
\hline C. nodulosa & CBS 160.58 & JN192383 & Eleusine indica \\
\hline C. oryzae & CBS $169.53^{\text {IsoT }}$ & КР400650 & Oryza sativa \\
\hline C. ovariicola & CBS $470.90^{\mathrm{T}}$ & MN688809 & Eragrostis interrupta \\
\hline C. pallescens & CBS $156.35^{\mathrm{T}}$ & KJ922380 & Air \\
\hline C. palmicola & MFLUCC $14-0404^{\mathrm{T}}$ & MF621582 & Acoelorrhaphe wrightii \\
\hline C. papendorfii & CBS $308.67^{\mathrm{T}}$ & KJ909774 & Acacia karroo \\
\hline C. paterae & CBS $198.87^{\mathrm{T}}$ & MN688810 & Triticum durum seed \\
\hline C. perotidis & CBS $350.90^{\mathrm{T}}$ & JN192385 & Perotis rara \\
\hline C. petersonii & BRIP $14642^{\mathrm{T}}$ & MH414905 & Dactyloctenium aegyptium \\
\hline C. portulacae & BRIP $14541^{\text {IsoT }}$ & KJ415553 & Portulaca oleracea \\
\hline C. pseudointermedia & CBS $553.89^{\mathrm{T}}$ & MN688819 & Cultivated pasture soil \\
\hline C. soli & CBS $222.96^{\mathrm{HT}}$ & KY905679 & Soil \\
\hline C. sorghina & BRIP $15900^{\mathrm{IsoT}}$ & KJ415558 & Sorghum bicolor \\
\hline C. sporobolicola & BRIP $23040^{\mathrm{HT}}$ & M̈H414908 & Sporobolus australasicus \\
\hline C. thailandica & MFLUCC $15-0747^{\mathrm{HT}}$ & MH275057 & Dead leaf of Pandanus sp. \\
\hline C. trifolii & CBS 173.55 & HG779023 & Trifolium repens \\
\hline C. tuberculate & CBS $146.63^{\text {IsoT }}$ & JX256433 & Zea mays \\
\hline C. xishuangbannaensis & MFLUCC $17-2271^{\mathrm{T}}$ & MH275058 & Dead leaf of Pandanus sp. \\
\hline Bipolaris maydis & CBS $136.29^{\mathrm{T}}$ & KJ909769 & Zea mays \\
\hline B. sorokiniana & CBS 110.14 & KJ922381 & Hordeum sp. \\
\hline \multicolumn{4}{|c|}{ DNA sequence accession numbers for phylogenetic analysis of Diaporthe } \\
\hline Diaporthe ambigua & CBS $114015^{\mathrm{T}}$ & KC343010 & Pyrus communis \\
\hline D. aquatica & IFRDCC $3051^{\mathrm{T}}$ & JQ797437 & Aquatic habitat \\
\hline D. brasiliensis & CBS $133183^{\mathrm{T}}$ & КС343042 & Aspidosperma tomentosum \\
\hline D. caatingaensis & CBS $141542^{\mathrm{HT}}$ & KY085927 & Tacinga inamoena \\
\hline D. citriasiana & ZJUD $30^{\mathrm{HT}}$ & JQ954645 & Citrus unshiu \\
\hline D. compacta & CGMCC $3.17536^{\mathrm{T}}$ & KР267854 & Camellia sinensis \\
\hline D. ganjae & CBS $180.91^{\mathrm{T}}$ & КС343112 & Cannabis sativa \\
\hline D. goulteri & BRIP 55657 $\mathrm{a}^{\mathrm{HT}}$ & KJ197290 & Helianthus annuus \\
\hline D. longispora & CBS $194.36^{\mathrm{T}}$ & КС343135 & Ribes sp. \\
\hline D. malorum & CAA734 ${ }^{\mathrm{HT}}$ & KY435638 & Malus domestica \\
\hline D. mayteni & CBS $133185^{\mathrm{HT}}$ & КС343139 & Maytenus acuminata \\
\hline D. neoraonikayaporum & MFLUCC $14-1136^{\mathrm{T}}$ & KU712449 & Tectona grandis \\
\hline D. oxe & CBS $133186^{\mathrm{T}}$ & КС343164 & Maytenus ilicifolia \\
\hline D. paranensis & CBS $133184^{\mathrm{T}}$ & КС343171 & Maytenus ilicifolia \\
\hline D. passifloricola & CBS $141329^{\mathrm{T}}$ & KX228292 & Passiflora foetida \\
\hline D. raonikayaporum & CBS $133182^{\mathrm{T}}$ & КC343188 & Spondias mombin \\
\hline
\end{tabular}


Table 1. Continued

\begin{tabular}{|c|c|c|c|}
\hline \multirow{2}{*}{\multicolumn{4}{|c|}{$\begin{array}{lcc}\text { Species } & \text { Isolates } & \text { ITS GenBank accession numbers } \\
\text { DNA sequence accession numbers for phylogenetic analysis of Diaporthe }\end{array}$}} \\
\hline & & & \\
\hline D. sclerotioides & CBS 296.67" & KC343193 & Cucumis sativus \\
\hline D. siamensis & MFLUCC $10-0573^{\mathrm{aT}}$ & JQ619879 & Dasymaschalon sp. \\
\hline \multirow[t]{2}{*}{ D. tectonae } & MFLUCC $12-0777^{\mathrm{HT}}$ & KU712430 & Tectona grandis \\
\hline & IPBCC 11.750 & SUB9431627 & Leaves of Guazuma ulmifolia \\
\hline D. tulliensis & BRIP $62248^{\text {ант }}$ & KR936130 & Theobroma cacao fruit \\
\hline D. corylina & CBS 121124 & КС343004 & Corylus sp. \\
\hline \multicolumn{4}{|c|}{ DNA sequence accession numbers for phylogenetic analysis of Didymella } \\
\hline Didymella acetosellae & CBS 179.97 & GU237793 & Rumex hydrolapathum \\
\hline D. aeria & LC $7441^{\mathrm{T}}$ & KY742051 & Air \\
\hline D. arachidicola & CBS 333.75"T & GU237833 & Arachis hypogea \\
\hline D. chloroguttulata & LC 7435 & KY742057 & Air \\
\hline \multirow[t]{2}{*}{ D. coffeae-arabicae } & CBS $123380^{\mathrm{HT}}$ & FJ426993 & Coffea arabicae \\
\hline & IPBCC 13.10895 & SUB9431855 & Leaves of Hydrocotyle verticillata \\
\hline D. dactylidis & CBS $124513^{\mathrm{HT}}$ & GU237766 & Dactylis glomerata \\
\hline D. exigua & CBS $183.55^{\mathrm{NT}}$ & GU237794 & Rumex arifolius \\
\hline D. glomerata & CBS 528.66 & FJ427013 & Chrysanthemum sp. \\
\hline D. longicolla & CBS $124514^{\mathrm{HT}}$ & GU237767 & Opuntia sp. \\
\hline D. molleriana & CBS 229.79 & GU237802 & Digitalis purpurea \\
\hline D. molleriana & CBS 109179 & GU237744 & Digitalis sp. \\
\hline D. ocimicola & LC 8137 & KY7420782 & Ocimum sp. \\
\hline D. pinodes & CBS 525.77ЕT & GU237883 & Pisum sativum \\
\hline D. protuberans & CBS 381.96 & GU237853 & Lycium halifolium \\
\hline Didymella pteridis & CBS 379.96 & KT389504 & Pteris sp. \\
\hline D. rhei & CBS 109177 & GU237743 & Rheum rhaponticum \\
\hline D. rumicicola & CBS 683.79"T & KT389503 & Rumex obtusifolius \\
\hline D. sancta & CBS 281.83 ${ }^{\mathrm{HT}}$ & FJ427063 & Ailanthus altissima \\
\hline D. senecionicola & CBS 160.78 & GU237787 & Senecio jacobaea \\
\hline D. suiyangensis & CGMCC $3.18352^{\mathrm{T}}$ & NR_158260 & Air \\
\hline \multirow[t]{2}{*}{ Phoma herbarum } & CBS $615.75^{\mathrm{T}}$ & KF251212 & Rosa multiflora cv. cathayensis \\
\hline & CBS 377.92 & KT389536 & Human leg \\
\hline \multicolumn{4}{|c|}{ DNA sequence accession numbers for phylogenetic analysis of Lasiodiplodia } \\
\hline Lasiodiplodia brasiliensis & CMM $4015^{\mathrm{HT}}$ & NR_147338 & Mangifera indica \\
\hline L. laelio-cattleyae & CBS $167.28^{\mathrm{T}}$ & MH854963 & Laelio cattleya \\
\hline L. citricola & IRAN1521 ${ }^{\mathrm{CT}}$ & GU945353 & Citrus sp. \\
\hline \multirow[t]{2}{*}{ L. crassispora } & CBS $118741^{\mathrm{T}}$ & DQ103550 & Santalum album \\
\hline & CMW 13488 & DQ103552 & Eucalyptus urophylla \\
\hline L. gilanensis & IRAN $1523^{\mathrm{CHT}}$ & GU945351 & Unknown \\
\hline L. jatrophicola & CMM $3610^{\mathrm{HT}}$ & NR_147348 & Jatropha curcas \\
\hline \multirow[t]{2}{*}{ L. margaritacea } & CBS $122519^{\mathrm{HT}}$ & EU144050 & Adansonia gibbosa \\
\hline & CBS 122065 & EU144051 & Adansonia gibbosa \\
\hline L. missouriana & CBS $128311^{\mathrm{HT}}$ & NR_145222 & Vitis sp. \\
\hline \multirow[t]{2}{*}{ L. gonubiensis } & CBS $115812^{\mathrm{T}}$ & DQ458892 & Syzigium cordatum \\
\hline & CBS 116355 & AY639594 & Syzigium cordatum \\
\hline L. magnoliae & MFLUCC 18-0948 & MK499387 & Magnolia candolii, dead leaves \\
\hline \multirow[t]{2}{*}{ L. mahajangana } & CBS $124925^{\mathrm{T}}$ & MH863425 & Terminalia catappa \\
\hline & IPBCC 11.751 & SUB 9431869 & Leaves of Guazuma ulmifolia \\
\hline L. parva & CBS $494.78^{\mathrm{T}}$ & EF622084 & Cassava-field soil \\
\hline L. pseudotheobromae & CBS $116459^{\mathrm{T}}$ & EF622077 & Gmelina arborea \\
\hline L. theobromae & CBS $164.96^{\mathrm{T}}$ & AY640255 & Fruit on coral reef coast \\
\hline \multirow[t]{2}{*}{ L. venezuelensis } & WAC12539нт & DQ103547 & Acacia mangium \\
\hline & WAC12540 & DQ103548 & Acacia mangium \\
\hline L. viticola & CBS $128313^{\mathrm{HT}}$ & MH864855 & $\begin{array}{l}\text { Wedge-shape canker of grapevine } \\
\text { cv. Vignoles }\end{array}$ \\
\hline Diplodia mutila & CBS 112553 & AY259093 & Vitis vinifera \\
\hline D. seriata & CBS $112555^{\mathrm{ET}}$ & AY259094 & Vitis vinifera \\
\hline
\end{tabular}


Table 1. Continued

\begin{tabular}{|c|c|c|c|}
\hline \multirow{2}{*}{\multicolumn{4}{|c|}{$\begin{array}{lcc}\text { Species } & \text { Isolates } & \text { ITS GenBank accession numbers } \\
\text { DNA sequence accession numbers for phylogenetic analysis of Neocosmospora }\end{array}$}} \\
\hline & & & \\
\hline Fusarium brasiliense & NRRL 31757 & EF408514 & Glycine $\max$ \\
\hline F. solani f. sp. batatas & NRRL 22400 & AF178407 & Ipomoea batatas \\
\hline F. solani f pisi & NRRL 22278 & DQ094309 & Pisum sativum \\
\hline F. solani f. sp. xanthoxyli & NRRL 22277 & AF178401 & Xanthoxylum sp. \\
\hline F. sriatum & NRRL 22101 & AF178398 & Cotton cloth \\
\hline Neocosmospora catenata & NRRL $54993^{\mathrm{HT}}$ & KC808256 & Zebra shark multiple tissues \\
\hline N. croci & CBS $142423^{\mathrm{HT}}$ & LT746264 & Citrus sinensis \\
\hline N. cyanescens & CBS $518.82^{\mathrm{T}}$ & EU329684 & Human foot \\
\hline N. falciformis & CBS $475.67^{\mathrm{T}}$ & MH859035 & Human bronchoalveolar lavage fluid \\
\hline N. gamsii & NRRL $32323^{\mathrm{HT}}$ & DQ094420 & Human bronchoalveolar lavage fluid \\
\hline N. illudens & NRRL 22090 & AF178393 & Beilschmiedia tawa \\
\hline N. lichenicola & NRRL 28030 & DQ094355 & Human \\
\hline \multirow[t]{2}{*}{ N. macrospora } & CBS $142424^{\mathrm{HT}}$ & LT746266 & Citrus sinensis \\
\hline & IPBCC 11.756 & SUB9433318 & Leaves of Centella asiatica \\
\hline N. mahasenii & CBS $119594^{\mathrm{HT}}$ & JF433045 & Dead branch of live tree \\
\hline N. petroliphila & NRRL 32315 & DQ094412 & Human groin ulcer \\
\hline N. plagianthi & NRRL 22632 & AF178417 & Hoheria glabrata \\
\hline \multirow{2}{*}{ N.pseudensiforme } & CBS $125729^{\mathrm{HT}}$ & KC691584 & Unknown dead tree \\
\hline & IPBCC 11.748 & SUB9431884 & Leaves of Guazuma ulmifolia \\
\hline N. solani & CBS $140079^{\mathrm{ET}}$ & KT313633 & Solanum tuberosum \\
\hline N. suttoniana & CBS $143214^{\mathrm{HT}}$ & DQ094617 & Human wound \\
\hline N. vasinfecta & CBS 130182 & EF453092 & Human \\
\hline Geejayessia cicatricum & CBS 125552 & HQ728145 & Buxus sempervirens \\
\hline G. atrofusca & NRRL 22316 & AF178423 & Staphylea trifolia \\
\hline \multicolumn{4}{|c|}{ DNA sequence accession numbers for phylogenetic analysis of Parengyodontium } \\
\hline Akanthomyces arachnophilus & BCC17655 & GQ249995 & Unknown \\
\hline A. novoguineensis & BCC22910 & GQ250003 & Insecta \\
\hline Beauveria amorpha & ARSEF $2641 \mathrm{~T}$ & NR_111601 & Hymenoptera: Formicidae \\
\hline B. caledonica & ARSEF 2567T & HQ880817 & Soil \\
\hline B. vermiconia & ARSEF 2922T & HQ880822 & Soil \\
\hline Cordyceps ninchukispora & BCC1422 & FJ765278 & Insecta \\
\hline C. pruinose & ARSEF 5413 & JN049826 & Iragoides fasciata (Lepidoptera) \\
\hline C. takaomontana & ВСС 1409 & EU807995 & Pupa \\
\hline Engyodontium parvisporum & IHEM 22910 & LC092896 & Indoor contamination \\
\hline E. rectidentatum & CBS 641.74 & LC092895 & Buried keratinous substance \\
\hline Isaria amoenerosea & CBS 107.73 & AY624168 & Coleopteran pupa \\
\hline I. cateniobliqua & CBS 153.83T & NR_111170 & Adoxophyes privatana \\
\hline I. cicadae & BCC 2574 & AY 624175 & Cicada nymph \\
\hline I. tenuipes & ARSEF 5135 & NR_119512 & Lepidopteran pupa \\
\hline Lecanicillium acerosum & CBS 418.81T & NR_111268 & Crinipellis perniciosa \\
\hline L. antillanum & CBS 350.85T & NR_111097 & Agaric \\
\hline L. aphanocladii & CBS 376.77 & AJ292431 & Agaricus bitorquis \\
\hline L. attenuatum & CBS $170.76 \mathrm{~T}$ & EF679164 & Caterpillar of Carpocapsa pomonella \\
\hline L. dimorphum & CBS 363.86T & NR_111101 & Agaricus bisporus \\
\hline L. flavidum & CBS 342.80T & NR_111266 & Decaying needle of Abies alba \\
\hline L. fungicola var. aleophilum & CBS 357.80T & NR_111064 & Agaricus bisporus \\
\hline L. fungicola var. fungicola & CBS 992.69T & NR_119653 & Agaricus bisporus \\
\hline L. indonesiacum & BTCC-F36T & АВ378516 & Araneae \\
\hline L. kalimantanense & BTCC-F23T & NR_121200 & Coleoptera in suspended soil \\
\hline L. longisporum & IMI 021167T & NR_111095 & Icerya purchasi (coccidae) \\
\hline L. nodulosum & IMI 338014R & EF513012 & Insect coccidae \\
\hline L. primulinum & JCM 18525T & NR_119418 & Soil \\
\hline L. saksenae & CBS 532.81T & JN049846 & Forest soil \\
\hline L. tenuipes & CBS 658.80 & LC092897 & Spider \\
\hline L. wallacei & CBS 101237T & NR_111267 & Lepidoptera \\
\hline \multirow[t]{2}{*}{ Parengyodontium album } & CBS 504.83ET & LC092880 & Human brain abscess \\
\hline & IPBCC 11.755 & SUB9446667 & Leaves of Guazuma ulmifolia \\
\hline
\end{tabular}


Table 1. Continued

\begin{tabular}{|c|c|c|c|}
\hline Species & Isolates & ITS GenBank accession numbers & Sources \\
\hline \multicolumn{4}{|c|}{ DNA sequence accession numbers for phylogenetic analysis of Parengyodontium } \\
\hline Simplicillium chinense & LC1345 & JQ410324 & Wood in freshwater \\
\hline S. cylindrosporum & JCM $18169^{\mathrm{T}}$ & NR_111023 & Soil \\
\hline S. lamellicola & CBS $116.25^{\mathrm{T}}$ & NR_111098 & Agaricus bisporus \\
\hline S. sympodiophorum & JCM $18184^{\mathrm{T}}$ & NR_111027 & Soil \\
\hline Torrubiella ratticaudata & ARSEF 1915 & JN049837 & Euophrys sp. \\
\hline Hypocrea lutea & GJS 89-129 & AF275339 & Decorticated conifer wood \\
\hline \multicolumn{4}{|c|}{ DNA sequence accession numbers for phylogenetic analysis of Talaromyces } \\
\hline Talaromyces amestolkiae & CBS $132696 \mathrm{HT}$ & JX315660 & House dust \\
\hline T. apiculatus & CBS 312.59HT & KF741983 & Soil \\
\hline T. aurantiacus & CBS 314.59HT & JN899380 & Soil \\
\hline T. coalescens & CBS $103.83 \mathrm{HT}$ & JN899366 & Soil under Pinus sp. \\
\hline T. diversus & CBS 320.48NT & KJ865740 & Leather \\
\hline T. duclauxii & CBS $322.48 \mathrm{HT}$ & JN899342 & Canvas \\
\hline T. echinosporus & CBS 293.62HT & JN899363 & Wood pulp \\
\hline T. erythromellis & CBS $644.80 \mathrm{HT}$ & JN899383 & Soil from creek bank \\
\hline T. marneffei & CBS 388.87T & JN899344 & Rhizomys sinensis \\
\hline T. minioluteus & CBS 642.68HT & JN899346 & Unknown \\
\hline T. muroii & CBS 756.96HT & JN899351 & Soil \\
\hline T. pittii & CBS 139.84NT & JN899325 & Clay soil under poplar trees \\
\hline T. purpureogenus & CBS 286.36HT & JX315671 & $\begin{array}{l}\text { Parasitic on a culture of Aspergillus } \\
\text { oryzae }\end{array}$ \\
\hline T. rademirici & CBS $140.84 \mathrm{NT}$ & JN899386 & Air under willow tree \\
\hline T. ruber & CBS 132704NT & JX315662 & Air craft fuel tank \\
\hline \multirow[t]{2}{*}{ T. trachyspermus } & CBS 373.48HT & JN899354 & Unknown \\
\hline & IPBCC 11.753 & SUB9431993 & Leaves of Guazuma ulmifolia \\
\hline T. varians & CBS 386.48HT & JN899368 & Cotton yarn \\
\hline Thermoascus crustaceus & CBS 181.67 & FJ389925 & Parthenium argentatum \\
\hline Muyocopron laterale* & IPBCC 13.1097 & SUB9857667 & Leaves of Hydrocotyle verticillata \\
\hline Speiropsis pedatospora* & IPBCC 11.754 & SUB9861920 & Leaves of Guazuma ulmifolia \\
\hline
\end{tabular}

Accession number of sequences obtained in this study are presented in italic

*Not included in the phylogenetic study

instead of endophytic fungus, while a $5 \mathrm{~mm}$ diameter culture plug of pathogenic fungi $F$. oxysporum was placed $1 \mathrm{~cm}$ away from the edge of the same Petri dish at the opposite side from the agar disc. All the plates were incubated at room temperature for seven days. The antagonistic activity was checked after incubation by measuring the growth radius of $F$. oxysporum on days 4 and 7 after inoculation. The magnitude of the inhibitory activity was calculated with the formula: $\mathrm{PI}=(100 \times(\mathrm{R} 1-\mathrm{R} 2) / \mathrm{R} 1)$, where PI is percentage inhibition of radial growth, $\mathrm{R} 1$ is the growth radius of $F$. oxysporum colony in the control plate, and R2 is radial growth of $F$. oxysporum in dual culture with the endophytic fungus. All of the endophytic fungi obtained in this study were tested, and each assay was repeated five times. Statistical analysis was done using the MSTAT program (University of WisconsinMadison), and mean values were analyzed by DMRT $(\mathrm{p}<0.05)$.

\section{Results}

\subsection{Diversity of Endophytic Fungi}

A total of 17 isolates of endophytic fungi having different colony characteristics were obtained from the four medicinal plants. Nine isolates of endophytic fungi were obtained from G. ulmifolia leaves, three isolates were obtained each from the leaves of $C$. asiatica and $H$. verticillata, and two isolates from the rhizomes of $C$. xanthorrhiza. Based on spore morphological characteristics, twelve of the fungal isolates could be classified into six genera, while the other five isolates were mycelia sterilia without spores. Leaves of $C$. asiatica were inhabited by Aspergillus, Colletotrichum, and Fusarium. Rhizomes of C. xanthorrhiza were inhabited by Aspergillus and Colletotrichum. Leaves of G. ulmifolia were occupied by Aspergillus, Colletotrichum, Curvularia, Fusarium, Phomopsis, Talaromyces, and three mycelia 
sterilia. Leaves of $H$. verticillata were occupied by Colletotrichum and two mycelia sterilia (Table 2).

Identification to species level with sequence analysis of ITS1-5.8S-ITS2 rDNA gave 16 good E value results out of 17 isolates (94.1\%), with the one remaining JBd11 isolate having poor quality DNA (Table 3). The JBd11 isolate was identified as Speiropsis pedatospora but with a low E value (6e-141). Based

Table 2. The morphological characteristics of the endophytic fungi associated with the medicinal plants

\begin{tabular}{ll}
\hline Medicinal plants & $\begin{array}{l}\text { Endophytic fungi/IPBCC } \\
\text { collection number }\end{array}$ \\
\hline C. asiatica & $\begin{array}{c}\text { Aspergillus sp.1 PLd3 } \\
\text { (IPBCC 11.760) }\end{array}$ \\
& $\begin{array}{l}\text { Colletotrichum sp.1 PLd6 } \\
\text { (IPBCC 13.1092) } \\
\text { Fusarium sp.1 PLd1 } \\
\text { (IPBCC 11.756) }\end{array}$
\end{tabular}

C. xanthorrhiza

Aspergillus sp.2 TLr5

(IPBCC 11.758)

Colletotrichum sp.2 TLr2 (IPBCC 11.757)

\section{G. ulmifolia}

\author{
Aspergillus sp.3 JBd3 \\ (IPBCC 11.749) \\ Colletotrichum sp.3 JBd1 \\ (IPBCC 11.747) \\ Curvularia sp. JBd14
(IPBCC 13.1088)
}

Fusarium sp.2 JBd2

(IPBCC 11.748)

Mycelia sterilia $1 \mathrm{JBd} 7$ (IPBCC 11.751)

Mycelia sterilia 2 JBd11 (IPBCC 11.754)

Mycelia sterilia 3 JBd13

(IPBCC 11.755)

Phomopsis sp.1 JBd4

(IPBCC 11.750)

Talaromyces sp. JBd10

(IPBCC 11.753)

H. verticillata

Colletotrichum sp.4 PBd3 (IPBCC 11.752)

Mycelia sterilia 4 PBd2 (IPBCC 13.10895)

Mycelia sterilia 5 PBd6 (IPBCC 13.1097)
Mycelium, conidiophore, and spore characteristics

Mycelium septate, conidiophores upright, simple, terminating in a globose bearing phialides at the apex, conidia 1 celled, globose, in dry basipetal chains, size 2.9-4.5 x 3.6-4.9 $\mu \mathrm{m}$

Mycelium septate, conidiophores simple, elongate, conidia hyaline, 1 celled, ovoid or oblong without appendages, size 17.5-23.2 x 5.9-6.5 $\mu \mathrm{m}$

Mycelium septate, conidiophores hyaline, slender and simple cell bearing phialides, conidia hyaline, two kinds, macroconidia several-celled, slightly curved with canoe-shaped, size 43.4-62.4 x 6.5-9.5 $\mu \mathrm{m}$, microconidia 1-2 celled, ovoid, oblong or slightly curved, size 10.3-16.6 x 3.4-4.6 $\mu \mathrm{m}$

Mycelium septate, conidiophores upright, simple, terminating in a globose bearing phialides at the apex, conidia 1 celled, globose, in dry basipetal chains, size 5.3-6.4 x 4.1-5.5 $\mu \mathrm{m}$

Mycelium septate, conidiophores simple, elongate, conidia hyaline, 1 celled, ovoid or oblong without appendages, size 5.3-11.4 x 4.1-5.5 $\mu \mathrm{m}$

Mycelium septate, conidiophores upright, simple, conidia 1 celled, globose, in dry basipetal chains, size 5.3-6.4 x 4.1-5.5 $\mu \mathrm{m}$

Mycelium septate, conidiophores simple, elongate, conidia hyaline, 1 celled, ovoid or oblong without appendages, size $20.8-29.3 \times 6.1-8.3 \mu \mathrm{m}$

Mycelium septate, conidiophores brown, simple, bearing spores apically or on new sympodial growing points, conidia dark, end cells lighter, $3-5$ celled, one of the central cells enlarged, size 37.4-65.3 x 15.1$18.2 \mu \mathrm{m}$

Mycelium septate, conidiophores hyaline, slender and simple cell bearing phialides, conidia hyaline, two kinds, macroconidia several-celled, slightly curved with canoe-shaped, size 34.0-59.9 x 4.6-6.8 $\mu \mathrm{m}$, microconidia 1 celled, ovoid, oblong or slightly curved, size 9.2-18.3 x 3.6-5.8 $\mu \mathrm{m}$

Mycelium septate, no conidia observed

Mycelium septate, no conidia observed

Mycelium septate, no conidia observed

Mycelium septate, conidiophores simple, conidia hyaline, 1 celled, size 10.8-15.2 x 4.1-6.7 $\mu \mathrm{m}$

Mycelium septate, conidiophores arising from the mycelium with verticilate bearing phialide, conidia hyaline, 1 celled, mostly ellipsoidal, size 7.3-8.6 x 6.9-8.3 $\mu \mathrm{m}$

Mycelium septate, conidiophores simple, elongate, conidia hyaline, 1 celled, ovoid or oblong without appendages, size 3.5-6.2 x 8.9-12.5 $\mu \mathrm{m}$

Mycelium septate, no conidia observed

Mycelium septate, no conidia observed 


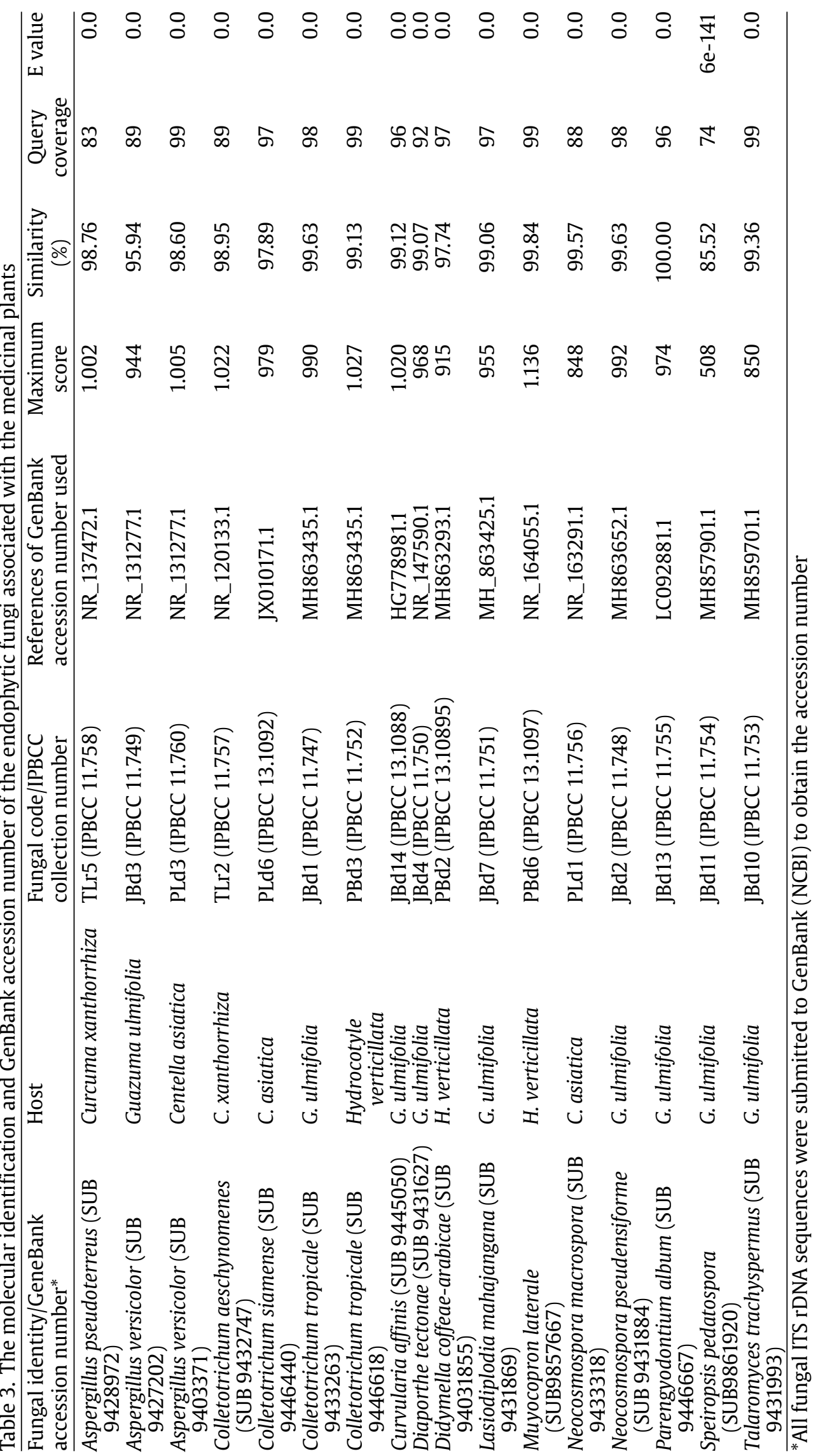


on BLAST analysis, the similarity of the isolated fungi to the closest species available in MycoBank and GenBank varied from $85.52 \%$ to $100.00 \%$, of which 13 isolates had a similarity value $>98 \%$. All of the isolates belong to Phylum Ascomycota in 3 classes, 8 orders, 11 families, and 15 identified species. The species are Aspergillus pseudoterreus, A. versicolor, Colletotrichum aeschynomenes, $C$. siamense, $C$. tropicale, Curvularia affinis, Diaporthe tectonae, Didymella coffeae-arabicae, Neocosmospora pseudensiforme, Lasiodiplodia mahajangana, Muyocopron laterale, Neocosmospora macrospora, Parengyodontium album, Speiropsis pedatospora, and Talaromyces trachyspermus (Table 3).

Fungal identification based on morphological characteristics uses microscopic observation of asexual spores (conidia). However, some isolates could not be differentiated based on morphological identification due to a lack of conidia. Mycelia sterilia 1 JBd7 and mycelia sterilia 3 JBd13 obtained from leaves of $G$. ulmifolia were identified as Lasiodiplodia mahajangana with $99.06 \%$ similarity and Parengyodontium album with $100.00 \%$ similarity. Similarly, mycelia sterilia 4 PBd2 derived from leaves of $H$. verticillata were successfully identified as Didymella coffeae-arabicae and mycelia sterilia 5 PBd6 was identified as Muyocopron laterale with $97.74 \%$ and $99.84 \%$ similarity to the sequences available in MycoBank, respectively (Table 2 and 3).

The JBd14 isolate was identified as Curvularia affinis with $99.12 \%$ similarity, and it was supported by phylogenetic analysis with $82 \%$ bootstrap support (Figure 1a). Based on sequence similarity comparison of the ITS region and phylogenetic analysis, $\mathrm{PBd} 2$ and JBd7 isolates were identified as Didymella coffeaearabicae and Lasiodiplodia mahajangana with $97.74 \%$ and 99.06\% similarity, respectively. Phylogenetic analysis showed that these isolates were in the same clade as those species with $>50 \%$ bootstrap support (Figure $1 \mathrm{~b}$ and $\mathrm{c}$ ). These three species of fungi belong to the Dothideomycetes class (https://www. mycobank.org).

The TLr5 isolate has sequence similarities of $98.76 \%$ with the species of $A$. pseudoterreus, and the isolate is in the same clade as this species with $98 \%$ bootstrap support (Figure 2a). The PLd3 isolate had relatively higher sequence similarities (98.6\%) with the species A. versicolor than with any other sequences, while the JBd3 isolate had sequence similarities at 95.94\%. In the phylogenetic tree, the two isolates were in one clade with $A$. versicolor (Figure 2a). Based on sequence similarity comparison of the ITS region and phylogenetic analysis, the JBd10 isolate is identified as $T$. trachyspermus with $99.36 \%$ similarity, and the isolate is in the same clade as this species with $100 \%$ bootstrap support (Figure 2b). These two species of fungi belong to the Eurotiomycetes class (https:// www.mycobank.org).

JBd 1 and TLr2 isolates were identified as C. tropicale with a relatively high homology value of $>98 \%$, while PLd6 and PBd3 were identified as C. siamense and $C$. aeschynomenes, respectively. Further analysis by phylogenetic tree showed that these isolates formed a sister clade with $88 \%$ bootstrap support (Figure 3a). The JBd4 isolate was closer to D. tectonae with $99.07 \%$ similarity. The result was supported by phylogenetic analysis with $97 \%$ bootstrap support (Figure $3 \mathrm{~b}$ ). The ITS sequence of the JBd2 isolate had a high similarity (>99\%) to N. pseudensiforme CBS 125729. The result was supported by phylogenetic analysis with $78 \%$ bootstrap support (Figure 3c). The PLd1 isolate is closer to $N$. macrospora species with a relatively higher similarity value of $>99 \%$, and phylogenetic analysis shows that the isolate belongs to the $N$. macrospora CBS 142424 clade with 97\% bootstrap support (Figure $3 \mathrm{c}$ ). Based on sequence similarity comparison of the ITS region and phylogenetic analysis, the JBD13 isolate was identified as $P$. album and phylogenetic analysis showed that this isolate was in the P. album CBS 504.83 clade with 99\% bootstrap support (Figure 3d). All four endophytic fungal genera belong to the Sordariomycetes class (https://www.mycobank.org).

\subsection{Antifungal Activity of Endophytic Fungi}

All isolated endophytic fungi showed inhibition activity against $F$. oxysporum. The percentage of inhibition varied from 6.0 to $78.9 \%$, and the differences are statistically significant. The inhibition values of endophytic fungi derived from leaves of $C$. asiatica, $G$. ulmifolia, $H$. verticillata, and rhizomes of $C$. xanthorhiza against $F$. oxysporum ranged $28.3-40.2 \%, 6.0-78.9 \%, 22.9-$ $36.1 \%$, and $41.0-44.8 \%$, respectively. The endophytic fungi T. trachyspermus and Curvularia affinis derived from leaves of G. ulmifolia showed the highest biocontrol activities against $F$. oxysporum with values of $78.9 \%$ and $60 \%$ inhibition, respectively (Table 4 ). 


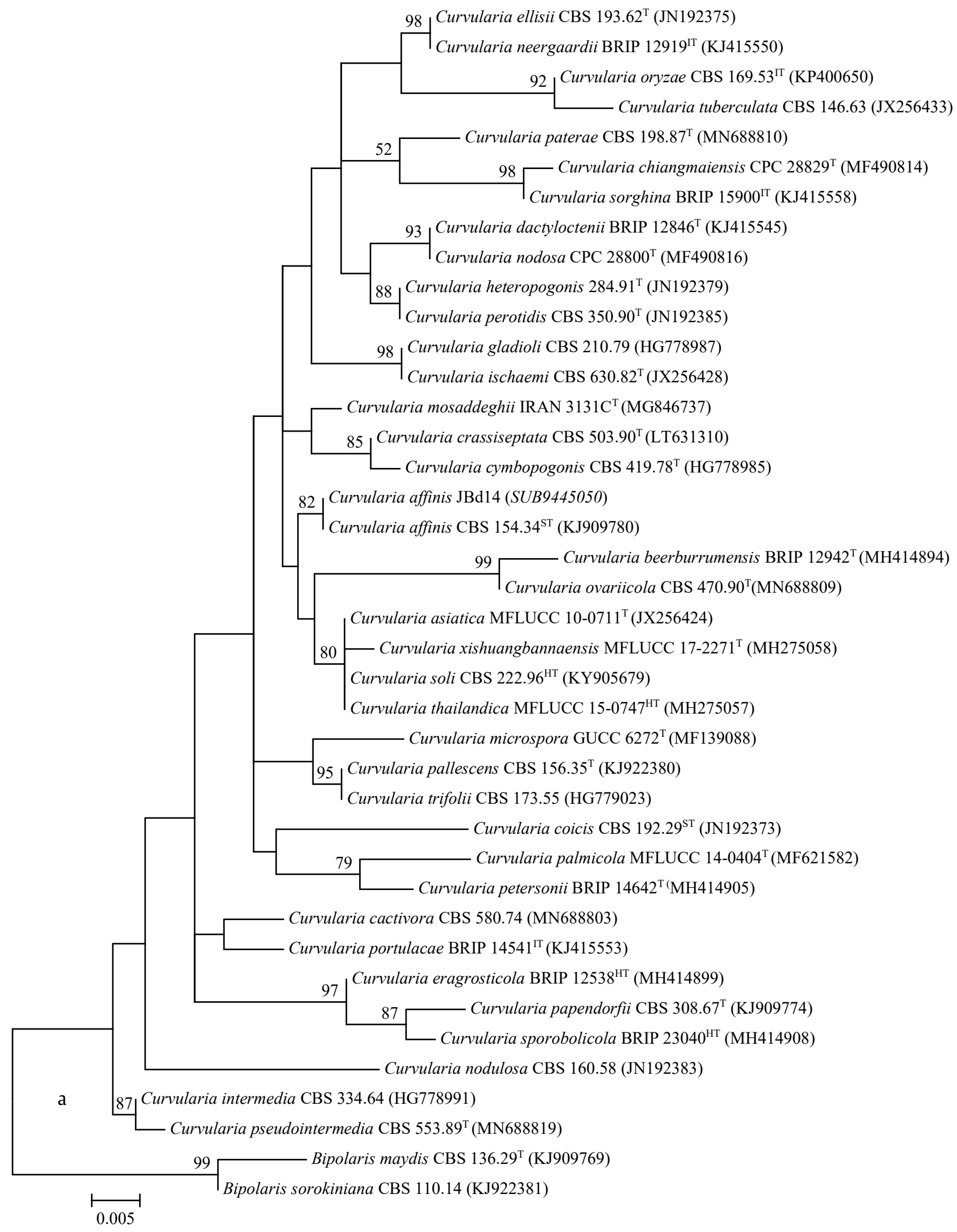




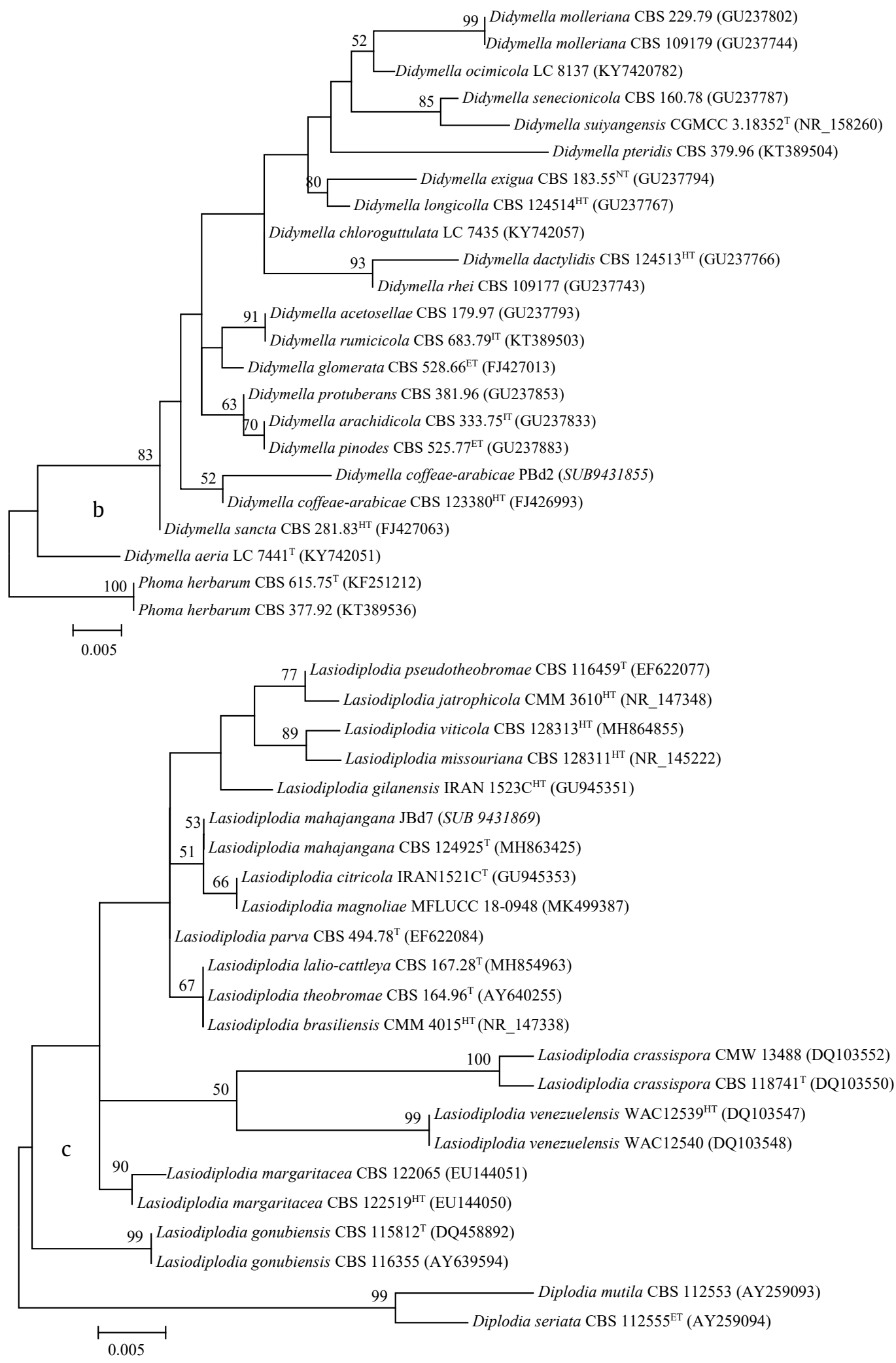

Figure 1. Maximum Likelihood phylogenetic analysis of ITS rDNA sequences of the Dothideomycetes endophytic fungi isolated from Guazuma ulmifolia and Hydrocotyle verticillata belonging to the genera (a) Curvularia, (b) Didymella, and (c) Lasiodiplodia. A phylogenetic tree was constructed using the MEGA ver. 6 program. Sequences obtained in the study are shown in bold. The marks ET, HT, IT, NT, T, and ST indicate epitype, holotype, isotype, neotype, type, and syntype strain, respectively. Numerical values $(>50)$ on branches are the bootstrap values as a percentage of bootstrap replication from a 1,000 replicate analysis 

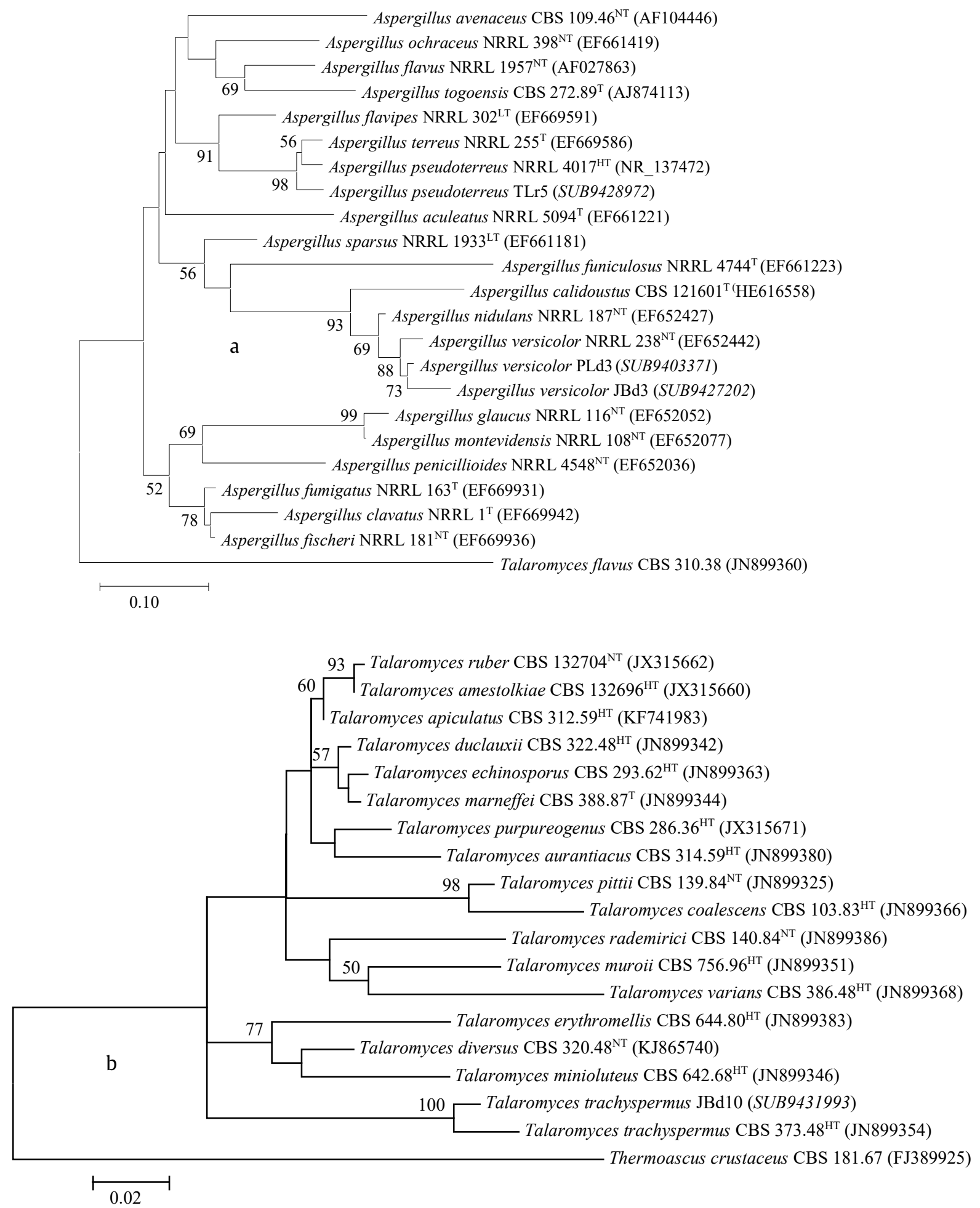

Figure 2.Maximum Likelihood phylogenetic analysis of ITS rDNA sequences of the Eurotiomycetes endophytic fungi isolated from Centella asiatica, Curcuma xanthorrhiza, and Guazuma ulmifolia that belonging to the genera (a) Aspergillus and (b) Talaromyces. A phylogenetic tree was constructed using the MEGA ver. 6 program. Sequences obtained in the study are shown in bold. The marks HT, LT, NT, and T indicate holotype, lectotype, neotype, and type strain, respectively. Numerical values $(>50)$ on branches are the bootstrap values as a percentage of bootstrap replication from a 1,000 replicate analysis 

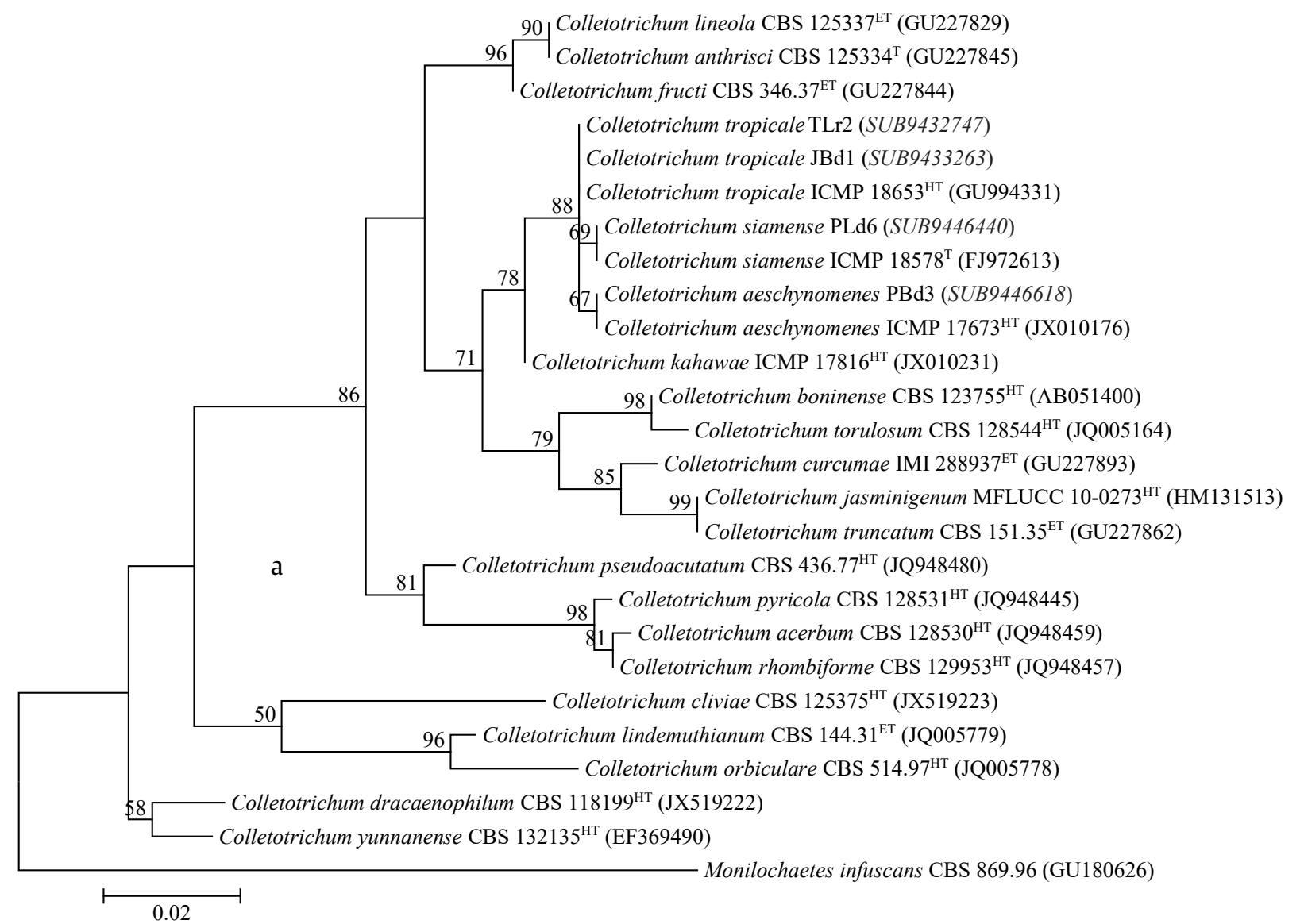

Monilochaetes infuscans CBS 869.96 (GU180626)

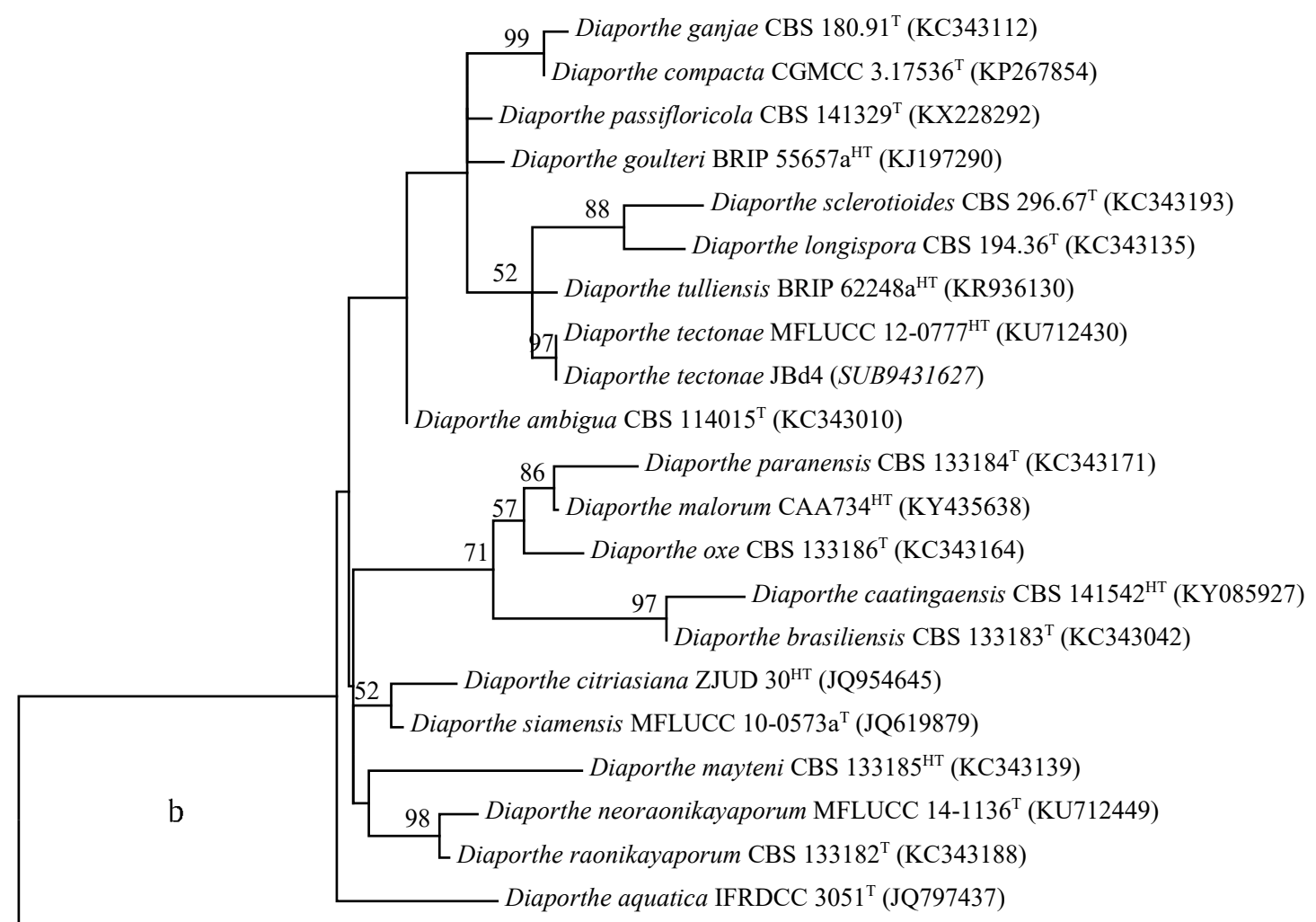

Diaporthella corylina CBS 121124 (KC343004) 


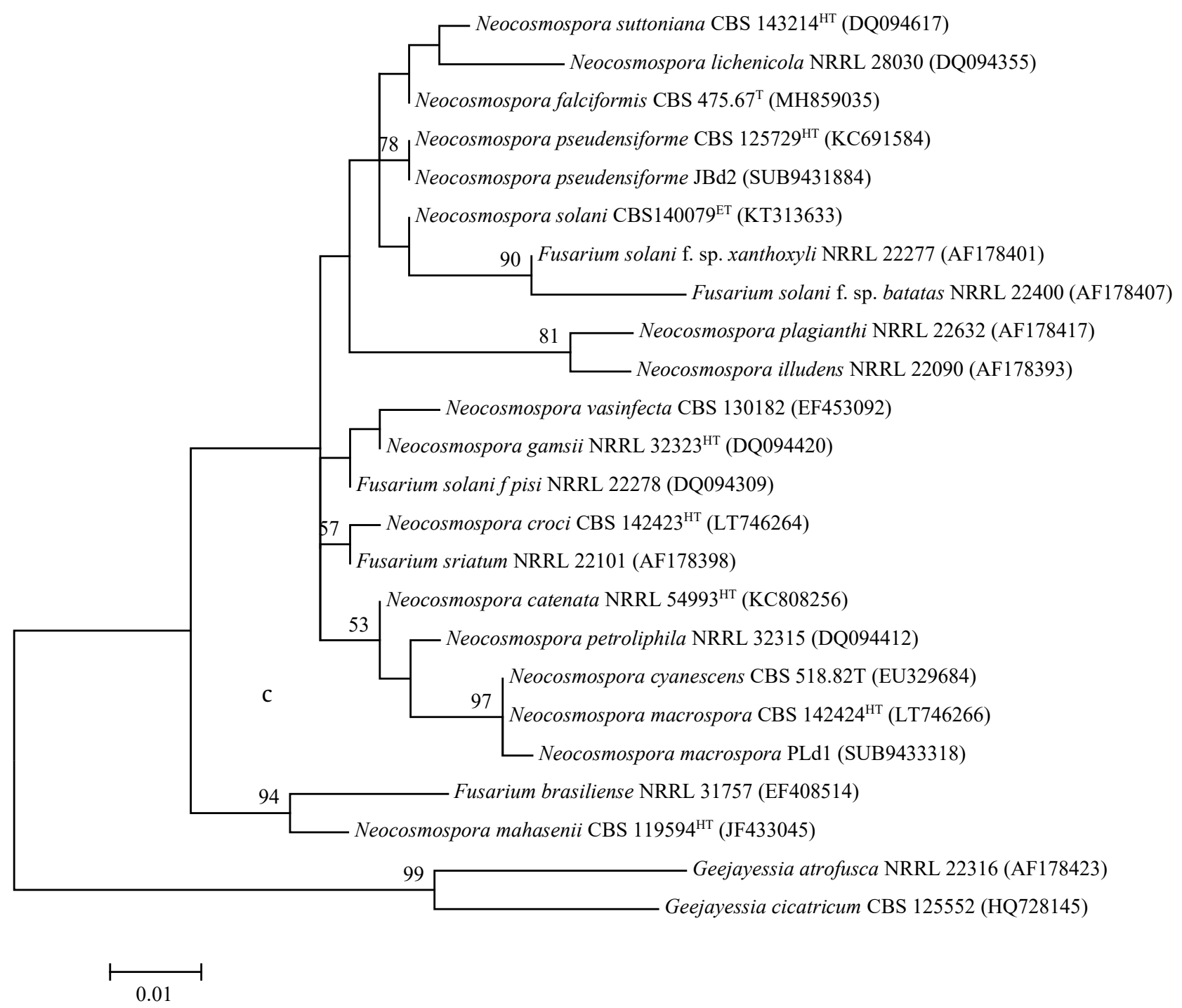




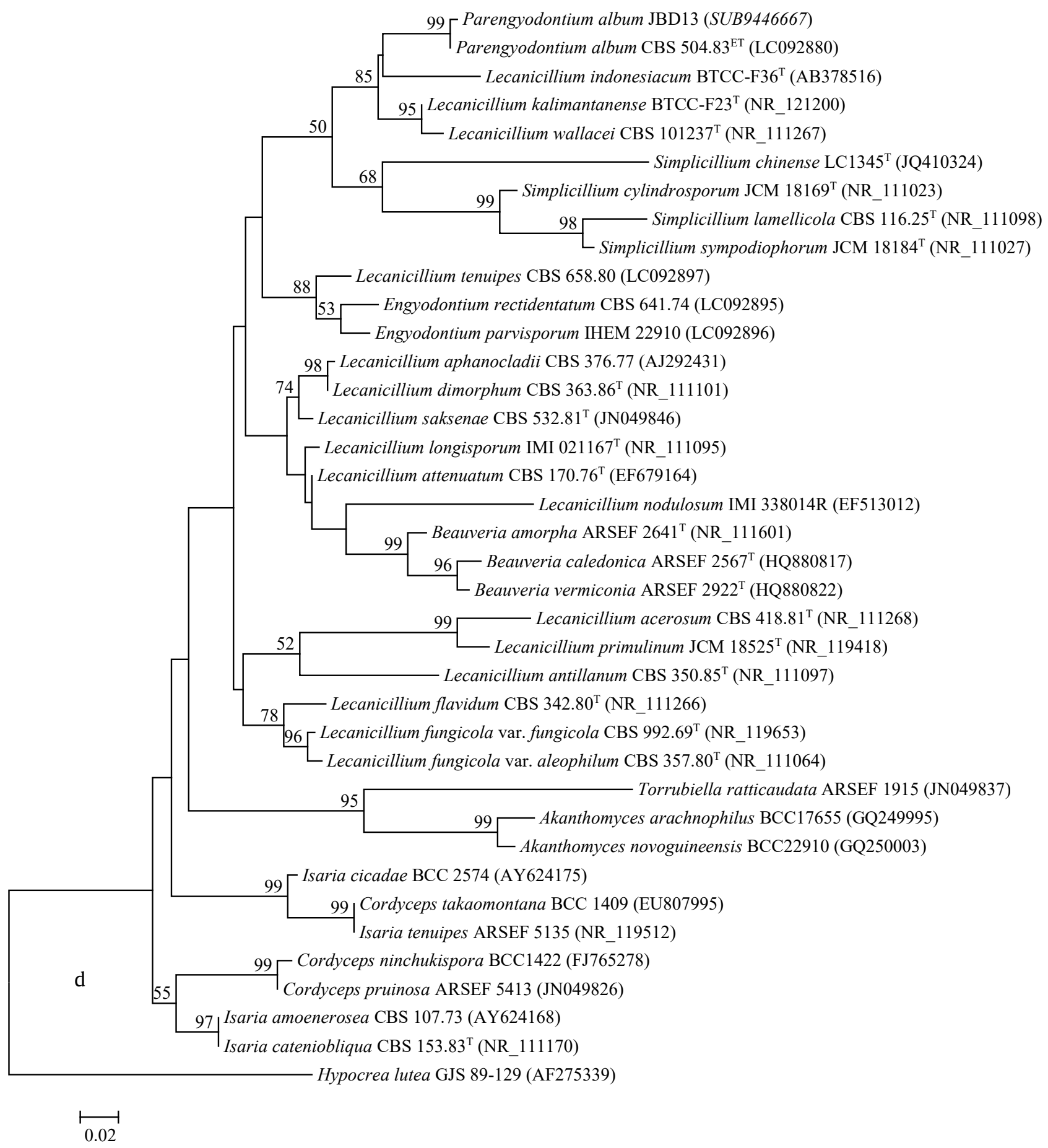

Figure 3.Maximum Likelihood phylogenetic analysis of ITS rDNA sequences of the Sordariomycetes endophytic fungi isolated from Centella asiatica, Curcuma xanthorrhiza, Guazuma ulmifolia, and Hydrocotyle verticillata belonging to the genera (a) Colletotrichum, (b) Diaporthe, (c) Neocosmospora, and (d) Parengyodontium. A phylogenetic tree was constructed using the MEGA ver. 6 program. Sequences obtained in the study are shown in bold. The marks ET, HT, and T indicate epitype, holotype, and type strain, respectively. Numerical values $(>50)$ on branches are the bootstrap values as a percentage of bootstrap replication from a 1,000 replicate analysis 
Table 4. Inhibition activity of fungal endophytes derived from the medicinal plants against Fusarium oxysporum

\begin{tabular}{llll}
\hline Endophytic fungi & $\begin{array}{l}\text { Fungal code/IPBCC } \\
\text { collection number }\end{array}$ & Host & \% Inhibition \\
\hline Aspergillus pseudoterreus & TLr5 (IPBCC 11.758) & Curcuma xanthorrhiza & $44.8 \mathrm{e}$ \\
Aspergillus versicolor & PLd3 (IPBCC 11.760) & Centella asiatica & $28.3 \mathrm{hi}$ \\
Aspergillus versicolor & JBd3 (IPBCC 11.749) & Guazuma ulmifolia & $41.2 \mathrm{f}$ \\
Colletotrichum aeschynomenes & TLr2 (IPBCC 11.757) & C. xanthorrhiza & $41.0 \mathrm{f}$ \\
Colletotrichum siamense & PLd6 (IPBCC 13.1092) & C. asiatica & $30.5 \mathrm{~h}$ \\
Colletotrichum tropicale & JBd1 (IPBCC 11.747) & G. ulmifolia & $49.1 \mathrm{c}$ \\
Colletotrichum tropicale & PBd3 (IPBCC 11.752) & Hydrocotyle verticillata & $36.1 \mathrm{~g}$ \\
Curvularia affinis & JBd14 (IPBCC 13.1088) & G. ulmifolia & $60.0 \mathrm{~b}$ \\
Diaporthe tectonae & JBd4 (IPBCC 11.750) & G. ulmifolia & $45.8 \mathrm{de}$ \\
Didymella coffeae-arabicae & PBd2 (IPBCC 13.10895) & H. verticillata & $25.9 \mathrm{ij}$ \\
Lasiodiplodia cit mahajangana & JBd7 (IPBCC 11.751) & G. ulmifolia & $48.1 \mathrm{~cd}$ \\
Muyocopron laterale & PBd6 (IPBCC 13.1097) & H. verticillata & $22.9 \mathrm{k}$ \\
Neocosmospora macrospora & PLd1 (IPBCC 11.756) & C. asiatica & $40.2 \mathrm{f}$ \\
Neocosmospora pseudensiforme & JBd2 (IPBCC 11.748) & G. ulmifolia & $25.0 \mathrm{jk}$ \\
Parengyodontium album & JBd13 (IPBCC 11.755) & G. ulmifolia & 6.01 \\
Speiropsis pedatospora & JBd11 (IPBCC 11.754) & G. ulmifolia & $40.3 \mathrm{f}$ \\
Talaromyces trachyspermus & JBd10 (IPBCC 11.753) & G. ulmifolia & $78.9 \mathrm{a}$ \\
\hline
\end{tabular}

avalues of \% inhibition are means from 5 replications, means followed by the same letter are not significantly different in $\operatorname{DMRT}(\mathrm{p}<0.05)$

\section{Discussion}

\subsection{Diversity of Endophytic Fungi}

All medicinally used organs of the plants studied were associated with endophytic fungi. The genus Colletotrichum was the most frequent endophyte found in this study and occupied all of the plants. The species $C$. aeschynomenes was found in leaves of $C$. xanthorrhiza, $C$. siamense was found in leaves of C. asiatica, and C. tropicale was found in leaves of $G$. ulmifolia and $H$. verticillata. The genus Colletotrichum is a ubiquitous endophyte and was isolated from roots, stems, branches, petioles, leaves, flowers, veins, bark, twig bark, twig xylem, intervein, and phloem of 73 medicinal plants (Rai et al. 2014). The genus Colletotrichum is reported as the dominant fungal endophyte in 16 out of 29 traditional Chinese medicinal plants (Huang et al. 2008) and Zingiber officinale (Ginting et al. 2013). The genus Aspergillus occupied all plants studied except $H$. verticillata. The fungus $A$. pseudoterreus was found to be associated with $C$. xanthorrhiza, whereas $A$. versicolor was associated with $C$. asiatica and G. ulmifolia in this study. The fungus Neocosmospora was found to be associated with G. ulmifolia and C. asiatica with different species for each plant. The species $N$. pseudensiforme was associated with G. ulmifolia, while $N$. macrospora was associated with $C$. asiatica in this study.
Some endophytic fungi are associated with specific hosts, and some species are associated with more than one host (Suryanarayanan et al. 2002). In this study, C. affinis, D. tectonae, L. mahajangana, $P$. album, S. pedatospora, and T. trachyspermus were found only in G. ulmifolia; while D. coffeae-arabicae and $M$. laterale were found in $H$. verticillata (Table 3 ). Host-specificity, host-recurrence, host selectivity, or host-preference is the relationship of fungal endophytes with single or multiple plant hosts (Cohen 2006). Some endophytic fungi are even tissued specific, fungi of different species occupy different tissues of a single plant (Ganley and Newcombe 2006).

\subsection{Antifungal Activity of Endophytic Fungi}

The inhibition activity of endophytic fungi against $F$. oxysporum can be grouped into low, medium, and high inhibition activity. Low inhibition activity was represented by the inhibition activity of $<30 \%$, the moderate inhibition activity by $30 \%$ to $59 \%$, and the high inhibition by $>60 \%$ (Table 4 ). From these isolates, five isolates (29.4\%) showed low, 10 isolates (58.8\%) showed moderate, and two isolates (11.8\%) showed high inhibition activity against $F$. oxysporum. The endophytic fungi obtained from G. ulmifolia ranged from low to high inhibition activity. The isolates derived from rhizomes of $C$. xanthorrhiza showed moderate inhibition activity, 
and the isolates from leaves of $C$. asiatica and $H$. verticillata showed low to moderate activity against F. oxysporum. The endophytic fungi T. trachyspermus JBd10 and C. affinis JBd14 derived from leaves of $G$. ulmifolia showed high inhibition activity. Strobel et al. (2007) reported that Muscodor albus isolated from G. ulmifolia produced unusual biochemical and biological properties.

The endophytic fungi $T$. trachyspermus and $C$. affinis derived from leaves of $G$. ulmifolia showed the highest biocontrol activities against $F$. oxysporum. Chomcheon et al.(2010) reported that Talaromyces sp. derived from mangrove could produce antimicrobial metabolites (7-epiaustdiol, stemphyperylenol, and secalonic acid A) to control Pseudomonas aeruginosa. Talaromyces is a teleomorph stage of Penicillium. Devi et al. (2012) isolated endophytic Penicillium sp. from $C$. asiatica, and it produced alkaloids, phenols, flavonoids, tannin, and glycosides. Furthermore, Shiozawa et al. (1994) reported that the species $T$. trachyspermus SANK 12191 produced trachyspic acid, a new metabolite that inhibited tumor cell's heparanase. Curvularia affinis isolated from the stem of Zingiber officinale had high antagonistic activity against $F$. oxysporum with a percentage inhibition value of $68.8 \%$ (Ginting et al. 2013). Curvularia affinis isolated from soil could produce the secondary metabolites pyrenocine J, pyrenochaetic acid $D$, pyrenocine $A$, and pyrenochaetic acid $A$. The metabolite pyrenocine J showed cytotoxic activity against human hepatic cancer (Zhang et al. 2012). In symbiotic interactions inside the host plants, the role of endophytic fungi is to protect the host plants from fungal pathogenic attack by direct and indirect mechanisms. The direct mechanism occurs through interaction between endophytes with fungal pathogens occupying the ecological niche, while the indirect mechanism is by inducing plant resistance. In the direct mechanism, endophytic fungi produce antibiotics and lytic enzymes that suppress the growth or kill pathogens. Various reports have documented that endophytic fungi grown in synthetic medium produce secondary metabolites that are powerful against pathogenic bacteria and fungi, including plant fungal pathogens (Gunatilaka 2006). These endophytic fungi are potential sources of antifungal compounds, particularly for controlling F. oxysporum.

\section{Conclusion}

There were 9 isolates of endophytic fungi obtained from the leaves of G. ulmifolia, 3 isolates each from the leaves of $C$. asiatica and $H$. verticillata, and 2 isolates from the rhizomes of $C$. xanthorrhiza. The genus Colletotrichum occupied all of the plants studied. The fungi $C$. affinis, D. tectonae, $L$. mahajangana, $P$. album, T. trachyspermus, and $S$. pedatospora were found only in G. ulmifolia; while $D$. coffeae-arabicae and $M$. laterale were found only in $H$. verticillata. Endophytic fungi derived from medicinally used organs of G. ulmifolia, C. asiatica, $H$. verticillata, and $C$. xanthorrhiza had inhibition activity against $F$. oxysporum with inhibition values ranging $6.0-78.9 \%$. T. trachyspermus JBd10 and $C$. affinis JBd14 had the highest inhibition values.

\section{Acknowledgements}

The research was funded by the Indonesian Agency for Agricultural Research and Development through KKP3T program (agreement No: 1998.9/ LB.620/I.1/5/2011 and No: 1142/LB.620/I.1/3/2012) and Research Center for Bioresources and Biotechnology, Bogor Agricultural University-Nara Institute of Science and Technology for cooperation among Young Researcher. Part of this research was also partially funded by the Indonesian Ministry of Research, Technology and Higher Education Republic of Indonesia for the World Class Professor Program Scheme A (agreement No: 123.12/D2.3/KP/2018) to Tropical Biopharmaca Research Center IPB. We thank the Indonesian Medicinal and Aromatic Crops Research Institute, Bogor, Indonesia for providing the plant samples for fungal isolation.

\section{References}

Abdollahzadeh J et al. 2010. Phylogeny and morphology of four new species of Lasiodiplodia from Iran. Persoonia 25:1-10.

Adhikari M et al. 2015. Discovery of two new Talaromyces species from crop field soil in Korea. Mycobiology 43:402-407.

Barnett HL, Hunter BB. 1998. Illustrated Genera of Imperfect Fungi. 4th ed. USA: Prentice-Hall Inc.

Cannon PF et al. 2012. Colletotrichum-current status and future directions. Stud Mycol 73:181-213.

Chen Q et al. 2017. Didymellaceae revisited. Stud Mycol 87:105-159. 
Chomcheon Pet al. 2010. Curvularides A-E: antifungal hybrid peptide-polyketides from the endophytic fungus Curvularia geniculata. Chem Eur J 16:11178-11185.

Cohen SD. 2006. Host selectivity and genetic variation of Discula umbrinella isolates from two oak species: analyses of intergenic spacer region sequences of ribosomal DNA. Microb Ecol 52:463-469.

Cook RK. 1993. Making greater use of introduced microorganisms for biological control of plant pathogens. Annu Rev Phytopathol 31:53-80.

Devi NN et al. 2012. Phytochemical analysis and enzyme analysis of endophytic fungi from Centella asiatica. Asian Pac J Trop Biomed 2:1280-1284.

Dissanayake AJ et al. 2017. The current status of species in Diaporthe. Mycosphere 8:1106-1156.

Ganley RJ, Newcombe G. 2006. Fungal endophytes in seeds and needles of Pinus monticola. Mycol Res 110:318-327.

Ginting RCB et al. 2013. Diversity of endophytic fungi from red ginger (Zingiber officinale Rosc.) plant and their inhibitory effect to Fusarium oxysporum plant pathogenic fungi. Hayati J Biosci 20:127-137.

Gunatilaka AAL. 2006. Natural products from plant-associated microorganisms: distribution, structural diversity, bioactivity, and implications of their occurrence. $J$ Nat Prod 69:509-526.

Hall TA. 1999. BioEdit: a user-friendly biological sequence analysis program for windows 95/98/NT. Nucleic Acids Sym Ser 41:95-98.

Hallmann J et al. 2007. Isolation procedures for endophytic microorganisms. In: Schulz B, Boyle C, Sieber TN (Eds.). Soil biology Vol. 9. New York: Springer-Verlag Berlin Heidelberg. pp. 299-319.

Hammerschmidt L et al. 2015. Two new metabolites from the endophytic fungus Xylaria sp. isolated from the medicinal plant Curcuma xanthorrhiza. Tetrahedron Lett 56:1193-1197.

Hardoim PR et al. 2015. The hidden world within plants: ecological and evolutionary considerations for defining functioning of microbial endophytes. Microbiol Mol Biol Rev 79:293-320.

Huang WY et al. 2008. Biodiversity of endophyte fungi associated with 29 traditional Chinese medicinal plants. Fungal Divers 33:61-75.

Kusari P et al. 2013. Endophytic fungi harbored in Cannabis sativa L.: diversity and potential as biocontrol agents against host plant-specific phytopathogens. Fungal Divers 60:137-151.

Marin-Felix Y et al. 2020. Multi-locus phylogeny of the genus Curvularia and description of ten new species. Mycol Progress 19:559-588.

Mycobank database. 2021. Fungal Databases, Nomenclature and Species Banks. Available at https://www. mycobank.org. [Date accessed: 5 March 2021]

Morton DT, Stroube NH. 1955. Antagonistic and stimulatory effects of microorganisms upon Sclerotium rolfsii. Phytopathology 45:417-420.

Nei M, Kumar S. 2000. Molecular evolution and phylogenetics. New York: Oxford University Press.

Rai M et al. 2014. Multiple applications of endophytic Colletotrichum species occurring in medicinal plants. In: Gurib-Fakim A (Eds.). Novel plant bioresources: applications in food, medicine, and cosmetics. Chichester: Wiley. pp. 227-236.
Rakotoniriana EF et al. 2008. Endophytic fungi from leaves of Centella asiatica: occurrence and potential interactions within leaves. Antonie van Leeuwenhoek 93:27-36.

Russell JR et al. 2011. Biodegradation of polyester polyurethane by endophytic fungi. Appl Environ Microbiol 77:60766084.

Sambrook J, Russel DW. 2000. Molecular Cloning: A Laboratory Manual. 3rd ed. Vol 3. Cold Spring Harbor:Laboratory Press.

Samson RA et al. 2014. Phylogeny, identification, and nomenclature of the genus Aspergillus. Stud Mycol 78:141-173.

Sandoval-Denis M, Crous PW. 2018. Removing chaos from confusion: assigning names to common human and animal pathogens in Neocosmospora. Persoonia 41:109-129.

Scarpari M et al. 2020. Didymella corylicola sp. nov., a new fungus associated with hazelnut fruit development in Italy. Mycol Prog 19:317-328.

Septiana E et al. 2017. Endophytic fungi associated with turmeric (Curcuma longa L.) can inhibit histamineforming bacteria in fish. Hayati J Biosci 24:46-52.

Shaw JJ et al. 2015. Biosynthesis and genomic analysis of medium-chain hydrocarbon production by the endophytic fungal isolate Nigrograna mackinnonii E5202H. Appl Microbiol Biotechnol 99:3715-3728.

Shiozawa $\mathrm{H}$ et al. 1994. Trachyspic acid, a new metabolite produced by Talaromyces trachyspermus that inhibits tumor cell heparanase: taxonomy of the producing strain, fermentation, isolation, structural elucidation, and biological activity. J Antibiot 48:357-362.

Strobel GA et al. 2007. Muscodor albus E-6, an endophyte of Guazuma ulmifolia making volatile antibiotics: isolation, characterization, and experimental establishment in the host plant. Microbiology 153:2613-2620.

Strobel GA, Daisy B. 2003. Bioprospecting for microbial endophytes and their natural products. Microbiol Mol Biol Rev 67:491-502.

Suryanarayanan TS et al. 2002. Occurrence and distribution of fungal endophytes in tropical forests across a rainfall gradient. Can J Bot 80:818-826.

Tamura Ket al. 2013. Mega6: molecular evolutionary genetics analysis version 6.0. Mol Biol Evol 30:2725-2729.

Thompson JD et al. 1994. Clustal W: improving the sensitivity of progressive multiple sequence alignment through sequence weighting, position-specific gap penalties, and weight matrix choice. Nucleic Acids Res 22:46734680.

Tsang et al. 2016. Cutaneous hyalohyphomycosis due to Parengodontium album gen. et comb. nov. Med Mycol 54:699-713.

White TJ et al. 1990. Amplification and direct sequencing of fungal ribosomal RNA genes for phylogenetics. In: Innis MA, Gelfand DH, Sninsky JS, White TJ.(Eds).PCR Protocols: a Guide to Methods and Applications. New York: USA, Academic Press. pp. 315-322.

Zhang $\mathrm{H}$ et al. 2012. Two new metabolites from a soil fungus Curvularia affinis strain HS-FG-196. J Asian Nat Prod Res 14:1078-1083. 